\title{
Gene fitness landscape of group A streptococcus during necrotizing myositis
}

\author{
Luchang Zhu, ${ }^{1}$ Randall J. Olsen, ${ }^{1,2}$ Stephen B. Beres, ${ }^{1}$ Jesus M. Eraso, ${ }^{1}$ Matthew Ojeda Saavedra, ${ }^{1}$ Samantha L. Kubiak, \\ Concepcion C. Cantu, ${ }^{1}$ Leslie Jenkins, ${ }^{3}$ Amelia R. L. Charbonneau, ${ }^{4,5}$ Andrew S. Waller, ${ }^{4}$ and James M. Musser ${ }^{1,2}$
}

'Center for Molecular and Translational Human Infectious Diseases Research, Houston Methodist Research Institute, and Department of Pathology and Genomic Medicine, Houston Methodist Hospital, Houston, Texas, USA. ${ }^{2}$ Department of Pathology and Laboratory Medicine, Weill Medical College of Cornell University, New York, New York, USA. ${ }^{3}$ Department of Comparative Medicine, Houston Methodist Research Institute, Houston, Texas, USA. ${ }^{4}$ Animal Health Trust, Newmarket, Suffolk, United Kingdom. ${ }^{5}$ Department of Veterinary Medicine, University of Cambridge, Cambridge, United Kingdom.

Necrotizing fasciitis and myositis are devastating infections characterized by high mortality. Group A streptococcus (GAS) is a common cause of these infections, but the molecular pathogenesis is poorly understood. We report a genome-wide analysis using serotype M1 and M28 strains that identified GAS genes contributing to necrotizing myositis in nonhuman primates (NHP), a clinically relevant model. Using transposon-directed insertion-site sequencing (TraDIS), we identified 126 and 116 GAS genes required for infection by serotype M1 and M28 organisms, respectively. For both M1 and M28 strains, more than $\mathbf{2 5 \%}$ of the GAS genes required for necrotizing myositis encode known or putative transporters. Thirteen GAS transporters contributed to both M1 and M28 strain fitness in NHP myositis, including putative importers for amino acids, carbohydrates, and vitamins and exporters for toxins, quorum-sensing peptides, and uncharacterized molecules. Targeted deletion of genes encoding 5 transporters confirmed that each isogenic mutant strain was significantly $(P<0.05)$ impaired in causing necrotizing myositis in NHPs. Quantitative reverse-transcriptase PCR (qRT-PCR) analysis showed that these $\mathbf{5}$ genes are expressed in infected NHP and human skeletal muscle. Certain substrate-binding lipoproteins of these transporters, such as Spy0271 and Spy1728, were previously documented to be surface exposed, suggesting that our findings have translational research implications.

\section{Introduction}

Necrotizing fasciitis, commonly known as the flesh-eating disease, is an invasive infection with very high rates of human morbidity and mortality (1). In severe cases, contiguous muscle may be severely damaged, resulting in necrotizing myositis. Based on whether the cause is polymicrobial or monomicrobial, necrotizing fasciitis can be divided into type I (polymicrobial) and type II (monomicrobial) $(1,2)$. Group A streptococcus (GAS) is the primary cause of type II necrotizing fasciitis and has an average case fatality rate of $29 \%(1,3,4)$. The molecular pathogenesis processes at work in GAS necrotizing fasciitis and myositis are poorly understood, a lack of knowledge that has impeded development of new effective diagnostics and therapeutics.

GAS is a human-specific pathogen causing more than 700 million infections annually worldwide (5). GAS infections range from relatively benign pharyngitis, skin, and soft-tissue infections to life-threatening invasive diseases, such as necrotizing fasciitis and necrotizing myositis (6). No vaccine is currently available to

\section{Related Commentary: p. 516}

Authorship note: LZ and RJO contributed equally to this work. Conflict of interest: The authors have declared that no conflict of interest exists. License: Copyright 2019, American Society for Clinical Investigation. Submitted: September 18, 2018; Accepted: November 27, 2018 Reference information: J Clin Invest. 2019;129(2):887-901. https://doi.org/10.1172/JCl124994. prevent GAS infections. Decades of research have revealed some of the GAS molecules that contribute to the pathogenesis of necrotizing fasciitis or myositis, including $\mathrm{M}$ protein (7), extracellular cysteine protease streptococcal pyrogenic exotoxin B (SpeB) (8), hyaluronic acid capsule $(7,9)$, secreted esterase (Sse) (10), and cytotoxins NADase and streptolysin O (11-13). However, although the genome of GAS is relatively small ( 1800 genes) (14), current understanding of the molecular pathogenesis of GAS necrotizing fasciitis and myositis is limited.

High-throughput genome-wide screens based on transposon mutagenesis strategies are very useful in providing new information about the genetic basis of bacterial virulence. Technologies such as signature-tagged mutagenesis (STM), transposon site hybridization (TraSH), and transposon-sequencing (Tn-Seq) have been applied successfully to many bacterial pathogens to identify genes required for fitness under diverse in vivo and ex vivo conditions (15-28). In GAS, genome-wide transposon mutagenesis screens have been used to identify genes contributing to fitness during growth in human blood ex vivo, human saliva ex vivo, and mouse subcutaneous infections $(15,21,29,30)$. However, a genome-wide investigation of the GAS genes contributing to fitness in necrotizing myositis has not been undertaken.

Analysis of the molecular pathogenesis of GAS necrotizing myositis requires use of appropriate animal models. Toward this end, mouse and nonhuman primate (NHP) necrotizing myositis models have been developed that approximate this disease (8). Importantly, GAS is a human-specific pathogen. Some GAS vir- 
A

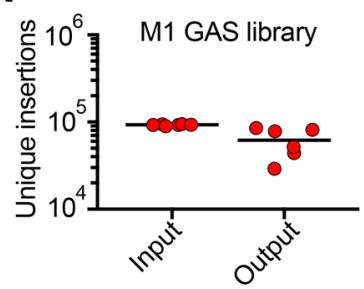

B
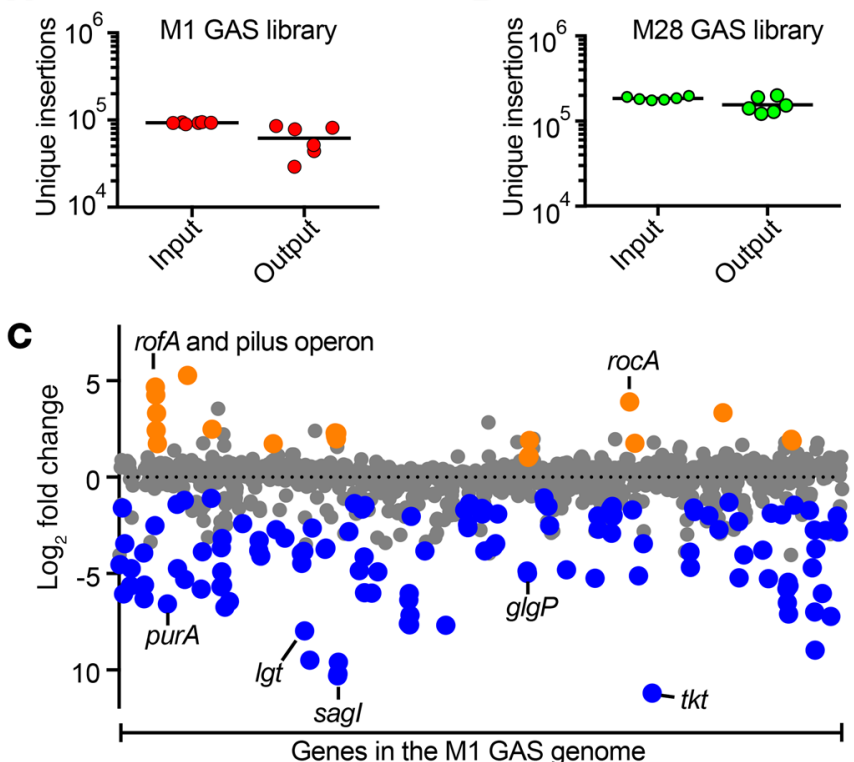

D

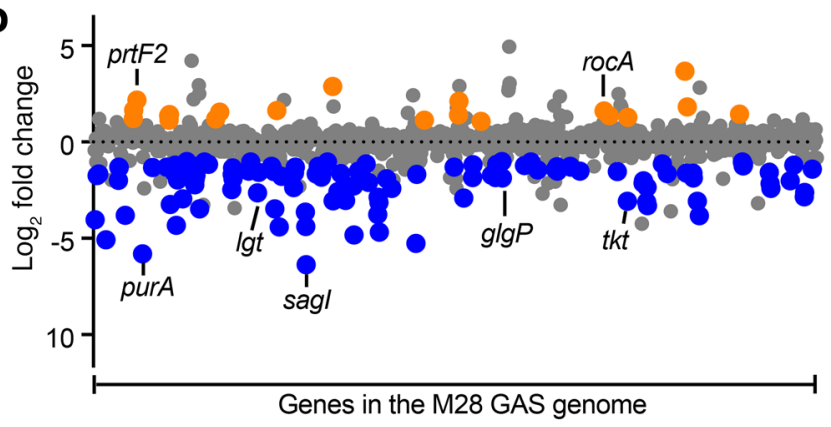

ulence factors are specific for human and NHP target molecules (31-34) and have significantly decreased or no activity against the mouse homologs (34-36). Thus, NHP necrotizing myositis provides the most relevant experimental model possible.

Here, we report what we believe is the first use in NHPs of a relatively new genome-wide transposon mutagenesis technique termed transposon-directed insertion-site sequencing (TraDIS) $(37,38)$. TraDIS was recently documented to provide much information about GAS genes contributing to fitness in human saliva (29). Using highly saturated transposon mutant libraries made in 2 genetically distinct GAS strains that are common causes of severe human infections, we identified genes required for bacterial fitness during necrotizing myositis. Our screen revealed that GAS transporters play a pivotal role in this infection. We confirmed and extended the TraDIS screen data using isogenic mutant strains, in vitro growth phenotyping, and quantitative reverse-transcriptase PCR (qRT-PCR) analysis of necrotic myositis tissues taken from infected NHPs and a human patient.

\section{Results}

Construction of highly saturated transposon mutant libraries in genetically representative strains of serotype $M 1$ and M28 of GAS. Transposon insertion mutant libraries were generated using serotype M1 strain MGAS2221 and serotype M28 strain MGAS27961 as the parental organisms. Strains of these $2 \mathrm{M}$ protein serotypes were
Figure 1. TraDIS analysis of GAS gene fitness in NHP necrotizing myositis. Complexity of the (A) M1 GAS mutant pools and (B) M28 CAS mutant pools before and after a 24-hour experimental NHP infection. Genomescale summary of the changes in mutant abundance ( $y$ axis) for each of the genes ( $x$ axis) in the (C) M1 GAS output pools and (D) M28 GAS output pools. Gene mutations (insertions) conferring significantly decreased (blue dots) or increased (orange dots) fitness are highlighted. Dots representing the gene mutations conferring significantly changed fitness are enlarged for visual emphasis.

used because they are among the 5 more abundant $\mathrm{M}$ protein types causing invasive infections in many countries, and in some cases they are the dominant causes of infections. Thus, serotype M1 and M28 GAS are clinically highly relevant (39-42). These 2 strains were chosen for transposon mutagenesis because (a) strain MGAS2221 is genetically representative of a pandemic serotype M1 clone that arose in the 1980s, rapidly spread worldwide, and currently is the most prevalent cause of severe infections globally (43-45), (b) strain MGAS27961 is genetically representative of a virulent serotype M28 clone that is prevalent in the United States and elsewhere (46), (c) both strains have WT alleles of all major transcriptional regulators that are known to affect GAS virulence, such as $\operatorname{cov} R$ and $\operatorname{covs}, \operatorname{rop} B, m g a$, and $\operatorname{roc} A$, and (d) both strains have been used previously in animal infection studies $(43,46)$. Using transposon plasmid pGh9:ISS1 (47), we generated dense transposon mutant libraries in strains MGAS2221 and MGAS27961 containing 154,666 (an insertion every 12 bp on average) and 330,477 (an insertion every 5.5 bp on average) unique transposon insertions, respectively (Supplemental Figure 1; supplemental material available online with this article; https://doi. org/10.1172/JCI124994DS1). This means that on average, the serotype M1 and M28 libraries had 66 and 139 insertions per open reading frame. The insertion index (number of unique insertions/ size of the gene) of each of the genes in the M1 and M28 genomes is illustrated in Supplemental Figure 1. Use of the MGAS2221 transposon mutant library to identify genes contributing to GAS fitness in human saliva ex vivo has been described recently (29).

Genome-wide screens identify GAS genes contributing to fitness in a necrotizing myositis infection model in cynomolgus macaques. We screened the M1 and M28 GAS transposon mutant libraries in NHPs as a first step toward discovering genes contributing to fitness during necrotizing myositis. Six cynomolgus macaques each were inoculated by intramuscular injection with either the M1 or M28 transposon mutant libraries and followed for 24 hours. All animals developed signs and symptoms consistent with necrotizing myositis and were necropsied. Biopsies containing necrotic muscle were obtained from the inoculation site to recover output mutant pools for subsequent analysis. Quantitative culture yielded an average of $4.87 \times 10^{8} \mathrm{CFU} / \mathrm{g}$ for M1 and $8.77 \times 10^{8}$ for M28 in the tissue biopsy specimens used for TraDIS analysis.

TraDIS was used to compare the mutant compositions of the input and output pools. The TraDIS analysis identified genes with significantly altered mutant frequency in the output mutant pools relative to the input mutant pool (examples shown in Supplemental Figure 2). Infection bottlenecks can be a technical challenge for high-throughput transposon mutagenesis studies, and substantial loss of mutant library complexity during animal infection 
A
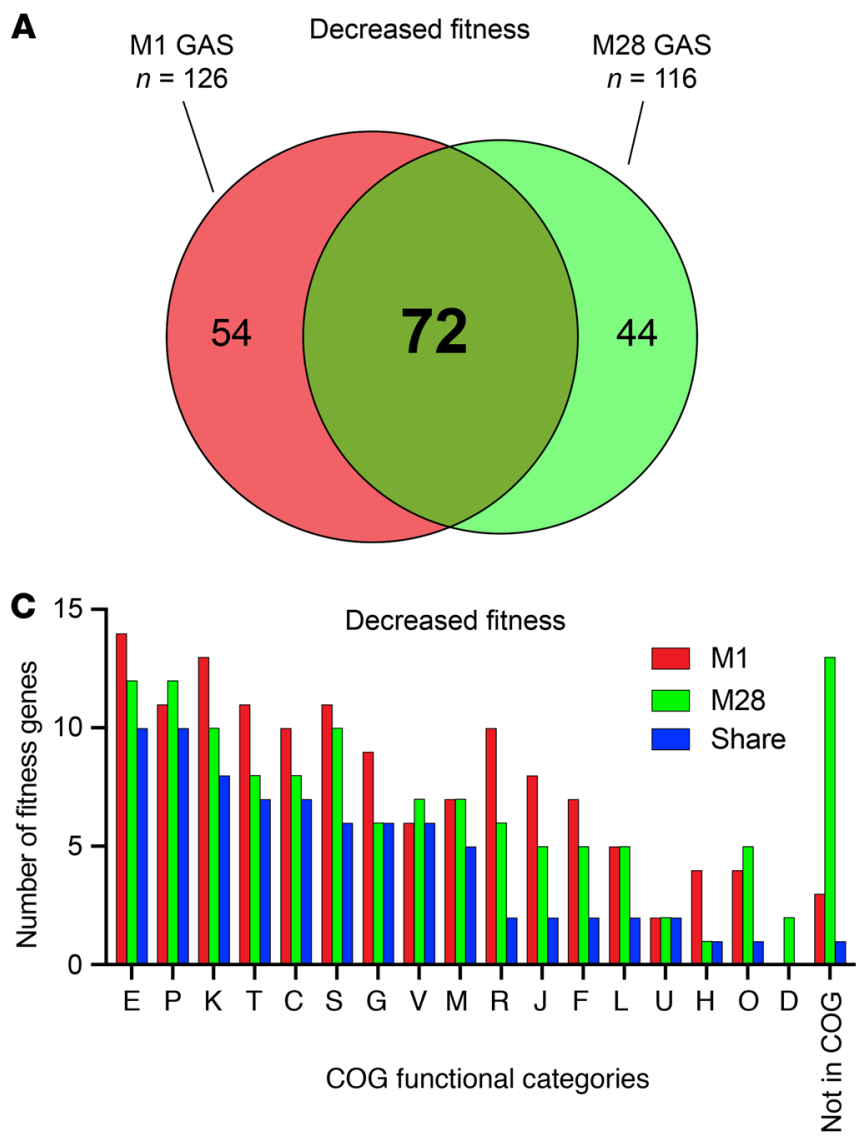

B Increased fitness

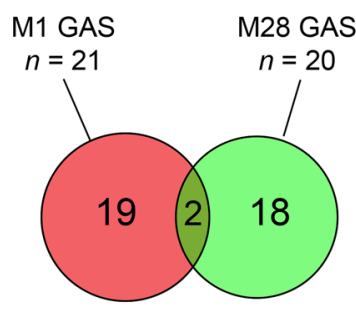

E Distribution in 62 sequenced GAS genomes

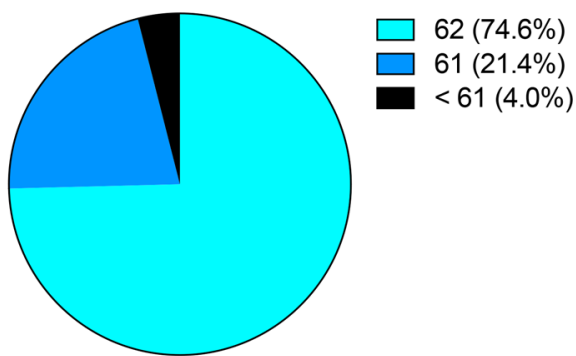

M1 GAS, decreased fitness Total $=126$

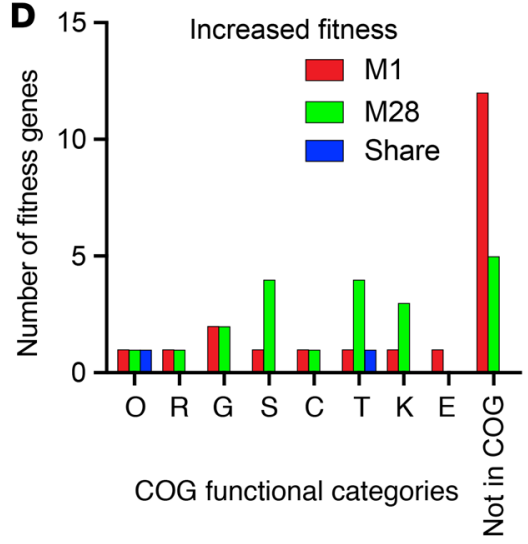

F Distribution in 62 sequenced GAS genomes

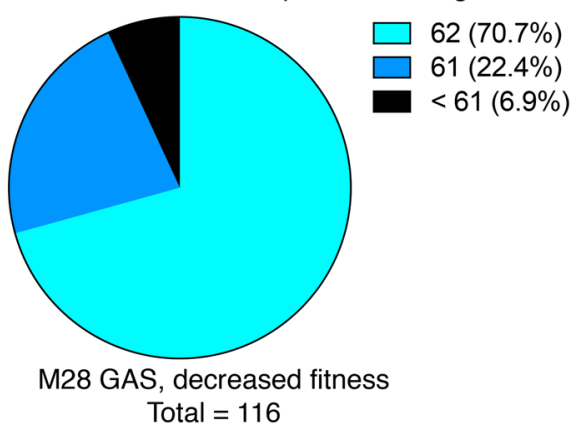

Figure 2. GAS gene mutations conferring significantly altered fitness during necrotizing myositis. Venn diagrams showing the number of mutated genes conferring significantly decreased fitness (A) and increased fitness (B) in M1 and M28 GAS strains during NHP infections. (C and D) Functional categorization of the identified GAS in vivo fitness genes during necrotizing myositis. (E and F) Distribution of the M1 and M28 CAS genes required for infection among the 62 sequenced GAS genomes.

can result in erroneous identification of fitness genes (48). Our TraDIS results showed that, for both M1 and M28 GAS screens, there was no substantial decline of mutant library complexity after NHP infections (Figure 1, A and B). On average, $67 \%$ and $84 \%$ of the library complexity remained in the M1 and M28 output pools, respectively (Figure 1, A and B). This high diversity of transposition site mutants recovered is inconsistent with a narrow infection bottleneck and indicates that our screens are unlikely to erroneously identify fitness genes. To identify GAS fitness genes in the infected NHP skeletal muscle milieu, genes previously identified as essential or critical for GAS growth in vitro in rich medium (THY) were excluded from the analysis (49) (Supplemental Table 1).
We also predicted GAS essential genes by analyzing the M1 and M28 GAS input libraries using the TraDIS toolkit (50) (Supplemental Tables 2 and 3). In general, the predicted M1 and M28 essential genes substantially overlapped with previously identified GAS genes essential or critical for growth in THY (49). Disrupted nonessential genes associated with significantly decreased fitness (transposon frequency $\log _{2}$ fold change $<-1 ; q<0.01$ ) in the output mutant pools were interpreted as contributing to NHP necrotizing myositis (Figure 1, C and D, and Figure 2). We identified 126 and 116 genes in the serotype M1 and M28 strains, respectively, that are crucial for GAS fitness in this infection model (Figure 2A), that is, inactivating these genes potentially confers diminished GAS 
A

M1 GAS, necrotizing myositis M1 GAS, saliva ex vivo
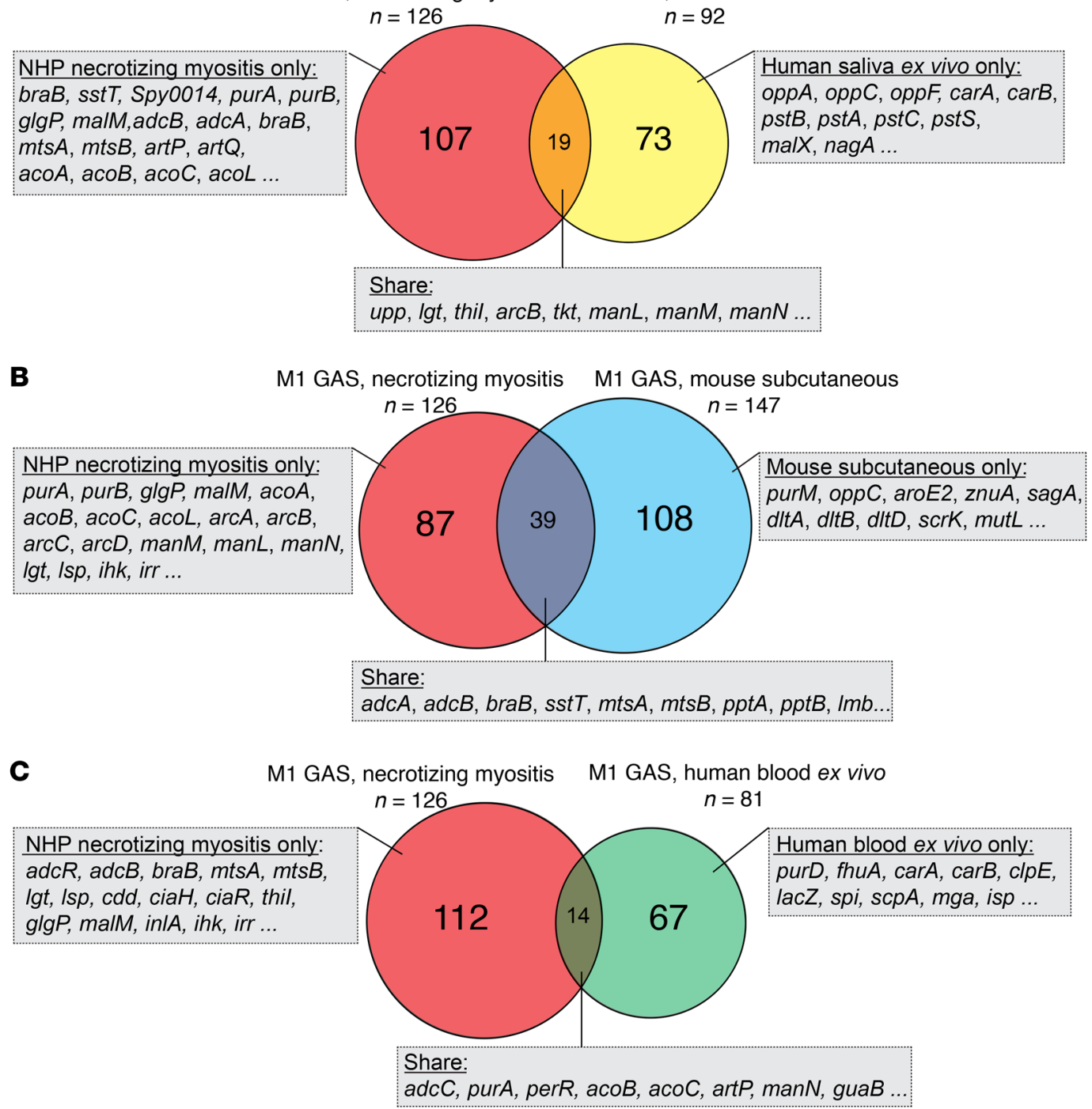

Figure 3. Lack of substantial overlap between GAS fitness genes required for necrotizing myositis and those required in other in vitro and in vivo environments. (A) Venn diagram comparison of 126 genes required for NHP necrotizing myositis with 92 genes required for optimal growth in human saliva ex vivo. (B) Venn diagram comparison of 126 genes required for necrotizing myositis with 147 genes required for mouse subcutaneous infection (30). (C) Venn diagram comparison of the 126 genes required for necrotizing myositis with 81 genes required for GAS growth in human blood ex vivo. Representative genes belonging to each category are listed in the shaded rectangular boxes (A-C).

fitness in necrotizing myositis. Importantly, a common set of 72 genes was identified as crucial for fitness in both the serotype M1 and M28 library NHP screens (Figure 2A and Supplemental Table 4). The shared 72 genes represent $57 \%$ of the serotype M1 fitness genes and $65 \%$ of the serotype M28 fitness genes (Figure 2A). Functional categorization of the fitness genes found that numerically, the more prevalent clusters of orthologous group (COG) (https://www.ncbi.nlm.nih.gov/COG/) categories included genes inferred to be involved in amino acid transport and metabolism (E), inorganic ion transport and metabolism (P), and transcription (K) (Figure 2C). Genes encoding many documented virulence factors or virulence-modulating factors were identified as contributing to fitness in both serotype M1 and M28 GAS strains, including $\operatorname{adcB} / C(51), \operatorname{gacI}(52,53)$, pepO $(54,55)$, inlA $(56)$, perR $(57)$, and $s c f A B(30)$ (Supplemental Tables 4, 5, and 7).
We also identified 21 and 20 genes in the serotype M1 and M28 strains, respectively, that were associated with significantly increased fitness in vivo (transposon insertion frequency $\log _{2}$ fold change $>1 ; q<0.01$ ) (Figure 2, B and D), that is, inactivating these genes potentially confers enhanced GAS fitness during NHP necrotizing myositis. These genes included known negative regulators of virulence $\operatorname{rivR}(58)$ and $\operatorname{roc} A$ (59-62). Of note, rocA and ppiB (peptidyl-prolyl cis-trans isomerase) were identified in both the serotype M1 and M28 strains (Figure 2B and Supplemental Tables 6 and 8). Inactivation of the rocA gene in an M28 GAS strain was recently shown to significantly increase virulence in a mouse model of necrotizing myositis (63).

To investigate the phylogenetic distribution of the identified in vivo fitness genes among diverse GAS strains, we examined the presence of M1 and M28 fitness genes required during NHP necro- 
A

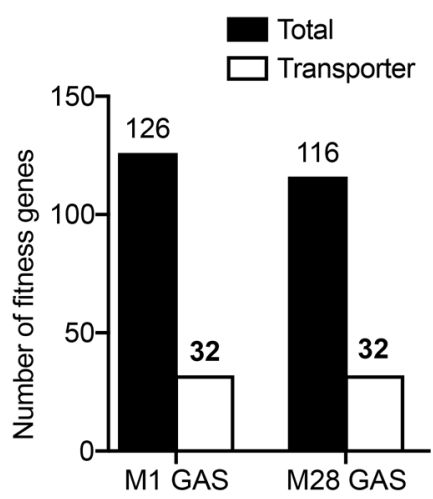

C

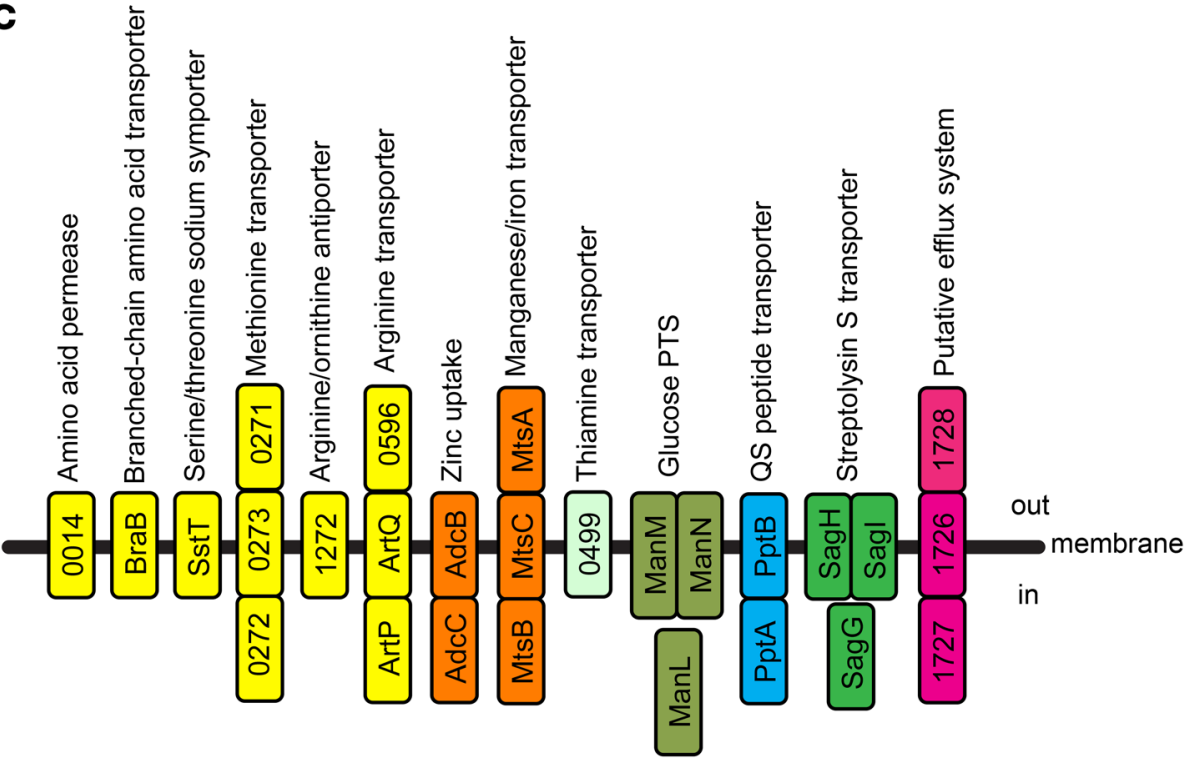

B M1 GAS transporter genes

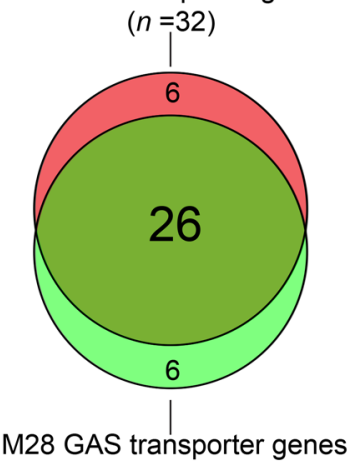

$(n=32)$

Figure 4. Genes encoding proven or putative transporters are an abundant portion of fitness genes that are required during necrotizing myositis in NHPs. (A) M1 CAS fitness genes $(n=32,25.4 \%)$ and M28 CAS fitness genes $(n=32,27.6 \%)$ that encode proven or putative transporters. (B) Venn diagram showing the relationship between $\mathrm{M} 1$ and $\mathrm{M} 28$ transporter genes required during NHP skeletal muscle infections; 26 genes are required in both M1 and M28 GAS strains. (C) Schematic showing the proven or putative transporters encoded by the shared transporter genes and their inferred functions. Inferred substrate-binding lipoproteins (Spy0271, Spy0596, MtsA, and Spy1728) are positioned outside of the bacterial cell. Elements that are positioned on the membrane and in the bacterial cell are putative transmembrane proteins and cytosolic proteins, respectively. The locus tag numbers refer to the annotation for serotype M1 GAS strain MGAS5005. PTS: phosphotransferase system; QS: quorum sensing.

tizing myositis in 62 sequenced GAS genomes representing 26 different M protein serotypes (Supplemental Table 9). The vast majority of the M1 fitness genes (96\%) and M28 fitness genes (93\%) were present in at least 61 of the 62 GAS strains (Figure 2, E and F).

Comparison of the genetic requirement for necrotizing myositis and those for GAS fitness in human saliva and blood ex vivo and mouse subcutaneous infections. Previous genome-wide transposon mutagenesis studies identified GAS genes required for growth in animal infection models and ex vivo in human body fluids such as blood and saliva $(21,29,30)$. These published data allowed us to test the hypothesis that the GAS gene requirements for NHP necrotizing myositis are distinct from those identified by transposon screens performed in other model infection environments, that is, we were able to assess the extent to which GAS has infection-specific genetic programs. We recently reported that 92 serotype M1 genes were required for optimal growth ex vivo in human saliva (29). Only 19 (21\% of 92) genes were defined as contributing to serotype M1 fitness in both human saliva and NHP necrotizing myositis (Figure $3 \mathrm{~A}$ ). The great majority of M1 genes $(n=107,85 \%)$ required in NHP necrotizing myositis did not overlap with fitness genes required ex vivo in human saliva. These genes included metabolic genes (purA, purB, acoABCL, $g l g P$, and $m a l M)$ and transporter genes ( $a d c A B, b r a B, m t s A, m t s B$, $\operatorname{art} P$, and $a r t Q)$ (Figure $3 A$ ). Using a similar transposon mutagen- esis technique (Tn-Seq), 147 genes were identified as contributing to fitness of serotype M1 GAS strain 5448 after subcutaneous inoculation in mice (30). The overlap between the mouse subcutaneous fitness genes and the 126 necrotizing myositis genes was relatively larger $(n=39)$ (Figure 3B). However, 69\% of the genes $(n=87)$ required for NHP necrotizing myositis did not overlap with the genes identified in the mouse subcutaneous infection study. These genes included metabolic genes purA, purB, acoABCL, glgP, malM, and $\operatorname{arcABCD}$ and phosphotransferase system gene manMLN (Figure $3 \mathrm{~B}$ ). Using a transposon mutagenesis technique, McIver and colleagues (21) identified 81 M1 GAS genes required for optimal bacterial growth in human blood. In comparison with the 126 necrotizing myositis fitness genes, only 14 genes were required in both conditions (Figure 3C), and $89 \%$ of the genes $(n=112)$ required for NHP necrotizing myositis did not overlap with the genes required in human blood ex vivo. These included genes for carbohydrate metabolism ( $g \lg P$, malM), transporters ( $a d c B, b r a B, m t s A, m t s B)$, and transcriptional regulators $(a d c R, c i a H, c i a R, i h k, i r r)$. To summarize, there is only modest overlap between the GAS genes contributing to fitness during experimental NHP necrotizing myositis relative to growth ex vivo in human saliva and blood and mouse subcutaneous infection. These results are consistent with our hypothesis that GAS has infection-specific genetic programs. 


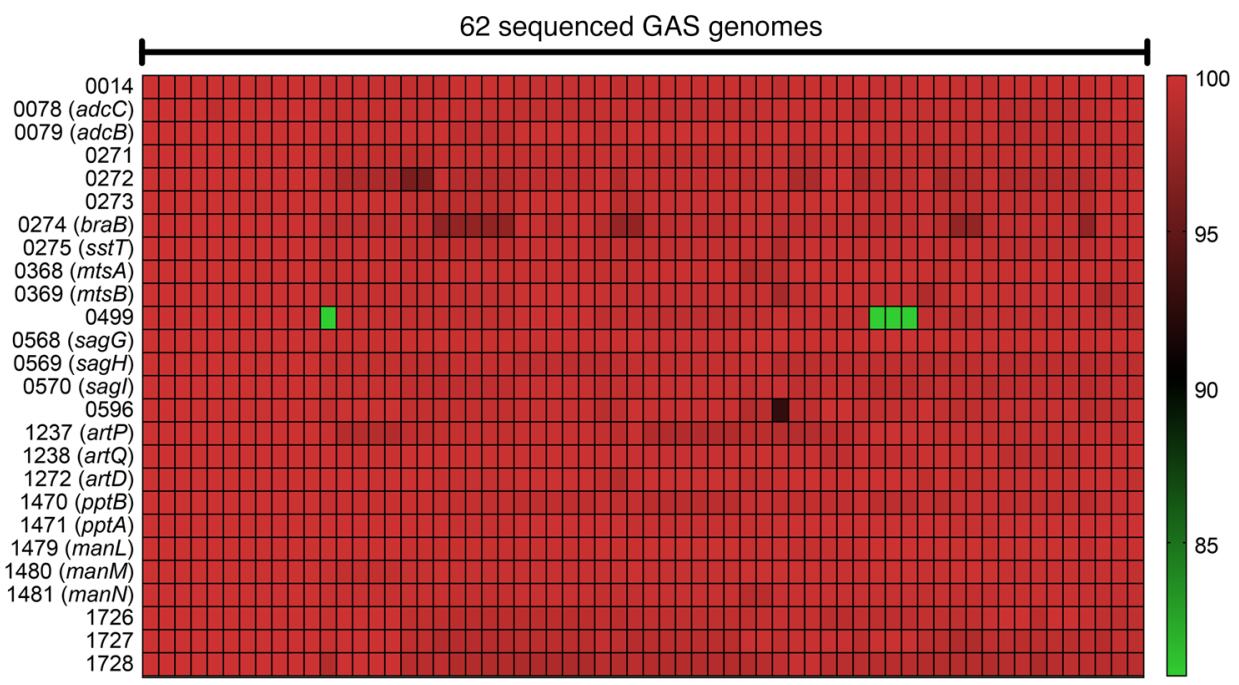

Figure 5. Conservation of the 26 GAS transporter genes among 62 sequenced GAS genomes. Heatmap showing the percentage identity of the 26 transporter genes of the 62 sequenced genomes (representing 26 different $\mathrm{M}$ protein serotypes) relative to those of the serotype M1 reference strain MGAS5005. The list of 62 sequenced genomes is included in Supplemental Table 9. The locus tag numbers refer to the annotation for serotype M1 CAS strain MCAS5005.

Genes encoding transporters constitute a considerable portion of GAS fitness determinants in experimental NHP necrotizing myositis. Bioinformatic analyses of the identified fitness genes found that, regardless of M1 or M28 serotype, more than 25\% of the genes contributing to in vivo fitness during NHP necrotizing myositis encoded proven or putative transporters (Figure 4A). Specifically, $25.4 \%$ of the serotype M1 fitness genes $(n=32)$ and $29.7 \%$ of the M28 GAS fitness genes $(n=32)$ encoded proven or putative transporters (Figure 4A). Importantly, 26 transporter genes are required in both M1 and M28 strains during infection, indicating that there was considerable overlap between the 2 sets of genes (Figure 4B). These 26 shared genes encoded 13 distinct transporters with proven or predicted roles in uptake of nutrients, such as amino acids, metal ions, vitamins, and carbohydrate, and export of a variety of substrates (Figure 4C). The DNA sequences of the 26 transporter genes were highly conserved (95\% to 100\% identity) among genomes for 62 sequenced GAS strains representing 26 different M protein serotypes (Figure 5). One gene (Spy0499) had less homology in GAS (81\% identical in 4 GAS strains) among the 62 strains with complete genomes.

Functional studies of genes encoding putative amino acid transporters. Six of the 13 transporters identified as important for both serotype M1 and M28 strains during NHP necrotizing myositis are putative amino acid transporters (Figure 4C). For example, Spy0014 is a putative amino acid permease and $\mathrm{BraB}$ is a putative branched-chain amino acid transporter. Spy0271, Spy0272, and Spy0273 constitute a putative ABC transporter similar to methionine transporter proteins MetQ (65\% identical), MetN (73\% identical), and MetP (71\% identical), respectively, of Streptococcus pneumoniae (64).

To test the hypothesis that Spy0014, BraB, and Spy0271-0273 participate in amino acid transport, we constructed isogenic deletion mutant strains $\triangle S p y 0014, \triangle b r a B$, and $\triangle S p y 0271-0273$ in parental M1 strain MGAS2221. We studied their growth phenotypes in rich medium (THY broth), and in a peptide-free chemically defined medium (CDM) (Figure 6). Compared with the WT parental strain, the 3 isogenic mutant strains did not exhibit a growth defect in THY medium (Figure 6A). However, the mutant strains had a severe growth defect when cultured in CDM (Figure 6B), a result consis- tent with our hypothesis. As anticipated, the growth defect of these 3 isogenic mutant strains, $\triangle S p y 0014, \Delta b r a B$, and $\Delta S p y 0271-0273$, was ameliorated by supplementing CDM with $0.1 \mathrm{~g} / \mathrm{ml}$ tryptone, a source of abundant peptides (Figure 6C). Together, these results are consistent with the idea that Spy0014, BraB, and Spy0271-0273 are amino acid transporters that are essential for GAS growth in the absence of a source of abundant exogenous peptides.

GAS is auxotrophic for 15 amino acids considered essential for growth $(65,66)$. We hypothesized that transporters Spy0014, BraB, and Spy0271-0273 contribute to uptake of specific essential amino acids. To test this hypothesis, we supplemented CDM with a high concentration $(1 \mathrm{~g} / \mathrm{l})$ of each of the highly soluble essential amino acids to determine whether certain amino acids restored the growth of these transporter mutants via nonspecific uptake (Figure 6, D-F). Consistent with the hypothesis, supplementing CDM with methionine restored the growth of mutant $\triangle$ Spy0271-0273 to near-WT growth phenotype, suggesting that Spy0271-0273 encodes a methionine transporter (Figure 6E). Similarly, supplementing CDM with histidine and valine partially restored the growth of mutant strains $\triangle S p y 0014$ and $\triangle b r a B$, respectively, suggesting that Spy0014 and BraB contribute to uptake of histidine and valine (Figure 6, D and F).

GAS genes required for growth in a peptide-free chemically defined medium. Many GAS fitness genes required during necrotizing myositis participated in amino acid uptake and were essential for GAS growth in CDM (Figures 4 and 6). This finding prompted us to systematically investigate GAS genes required for growth in CDM, where amino acid uptake is essential for growth. We exposed the M1 and M28 GAS transposon mutant libraries to CDM to screen for genes required for growth in this nutrient-poor medium. Using TraDIS, we identified 34 and 12 genes important for growth in CDM in M1 and M28 GAS strains, respectively (Figure 7 and Supplemental Tables 12 and 14). A common set of 6 genes encoding amino acid transporters (Spy0014, Spy0271, Spy0272, Spy0273, braB, and sstT) were identified as critical for growth in CDM in both M1 and M28 GAS. Importantly, these 6 amino acid transporters required for optimal GAS growth in CDM were also important for GAS fitness during necrotizing myositis (Figure 7, A and B). We also identified 21 and 4 genes in M1 and M28 GAS strains that, when mutated, conferred increased fit- 

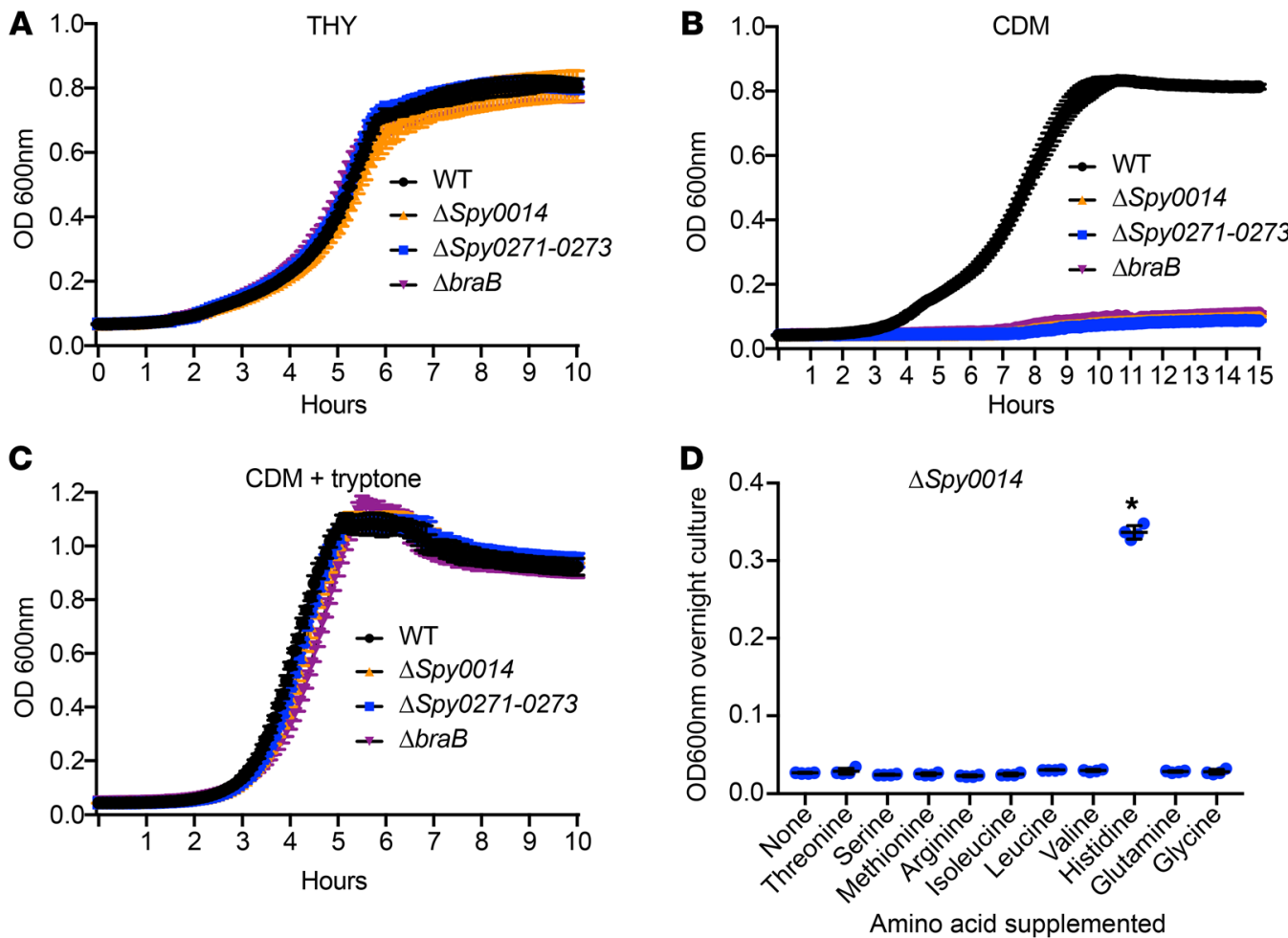

$\mathbf{F}$

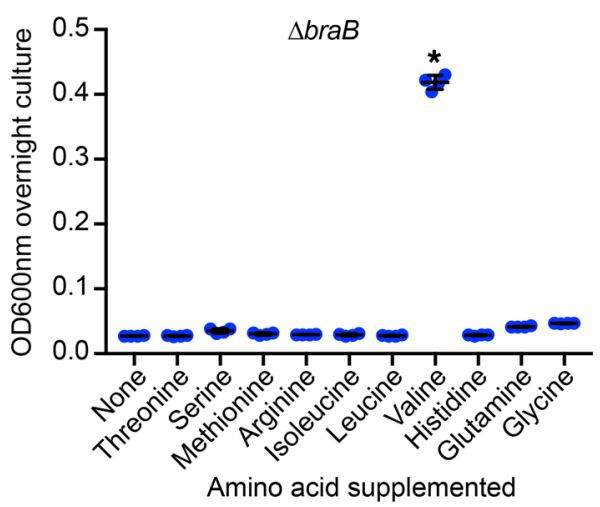

Figure 6. In vitro phenotype of $\mathbf{3}$ amino acid transporter mutant strains. (A-C) Growth of parental WT strain MGAS2221 (WT), $\triangle$ Spy0014, $\triangle b r a B$, and $\triangle$ Spy0271-0273 in rich medium THY (A), CDM (B), and CDM supplemented with $10 \mathrm{~g} / \mathrm{l}$ tryptone (C). (D-F) Growth of 3 mutant strains in CDM supplemented with $1 \mathrm{~g} / \mathrm{l}$ of specified amino acids. Replicate data $(n=4)$ are expressed as mean $\pm \mathrm{SD}$ in $\mathbf{D}, \mathbf{E}$, and $\mathbf{F}$. ${ }^{*} P<0.05$ vs. unsupplemented group, 1-way ANOVA.
E
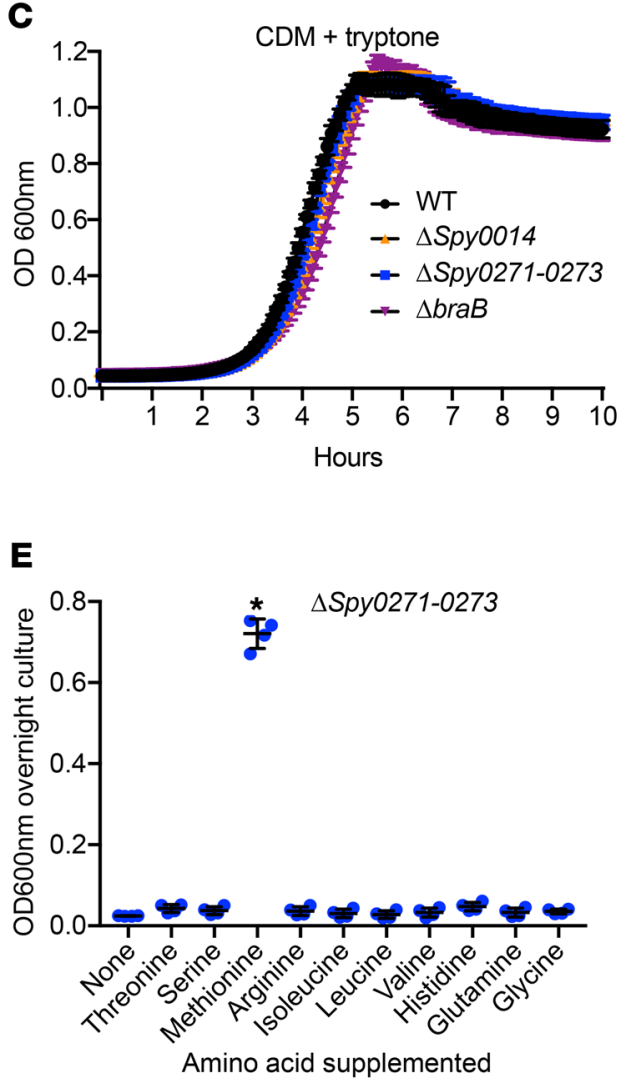

ness in CDM (Supplemental Tables 13 and 15). Collectively, our results show that amino acid transporters are crucial for GAS fitness in CDM in vitro and during necrotizing myositis.

Virulence role of genes encoding putative amino acid transporters. To validate the TraDIS screen results in vivo, we infected NHPs in the quadriceps with parental M1 strain MGAS2221, and isogenic mutant strains $\Delta S p y 0014, \Delta b r a B$, and $\Delta$ Spy0271-0273. Compared with the WT parental strain, each of these 3 transporter mutant strains caused significantly smaller lesions characterized by less tissue destruction in the NHP necrotizing myositis model (Figure $8, \mathrm{~A}, \mathrm{~B}, \mathrm{E}$, and F). In addition, compared with what occurred with the WT parental strain, significantly fewer CFUs of each isogenic mutant strain were recovered from the inoculation site and a distal site of dissemination (Figure 8, C and D).

Virulence role of Spy1726-1728, a poorly characterized ABC transporter. Our genome-wide screens identified a putative ABC transporter of unknown function that is required for NHP infection by the M1 and M28 GAS strains (Figure 4C). This putative transporter is encoded by 3 contiguous genes: Spy1726 (transporter permease protein), Spy1727 (ATP-binding protein), and Spy1728 (substrate-binding lipoprotein). To confirm the virulence role of this putative transporter, we used isogenic mutant strain $\triangle S p y 1726-1728$ made by deleting the entire Spy1726-1728 region in serotype M1 parental strain MGAS2221. Consistent with the result from the initial NHP necrotizing myositis TraDIS screen, isogenic mutant strain $\Delta S p y 1726-1728$ was significantly attenuated in its capacity of causing necrotizing myositis in NHPs (Figure 8, A-D). This putative ABC transporter operon was not identified as important for virulence in previous GAS transposon mutagenesis screens $(15,21,30)$, suggesting an infection- or primate-specific role in necrotizing myositis. Of note, Spy1728 (a substrate-binding lipoprotein) was previously shown to be a GAS surface protein and potential vaccine candidate (67).

Virulence role of quorum-sensing peptide transporter PptAB. Our genome-wide TraDIS screens suggested that inactivating the quorum-sensing peptide transporter PptAB in the serotype M1 and M28 strains resulted in significantly $(P<0.05)$ decreased fitness 
A

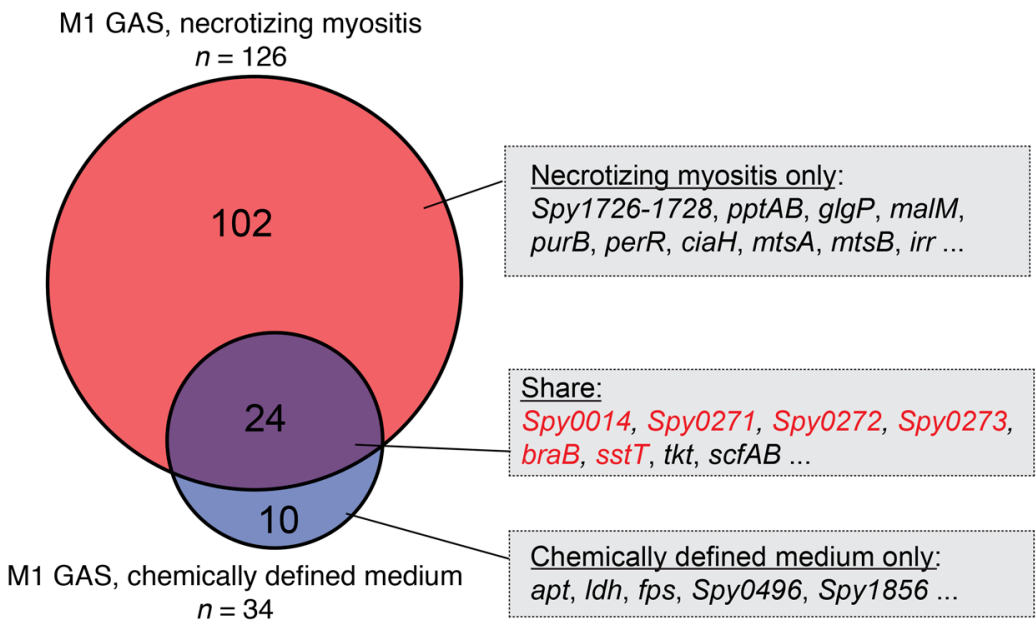

B

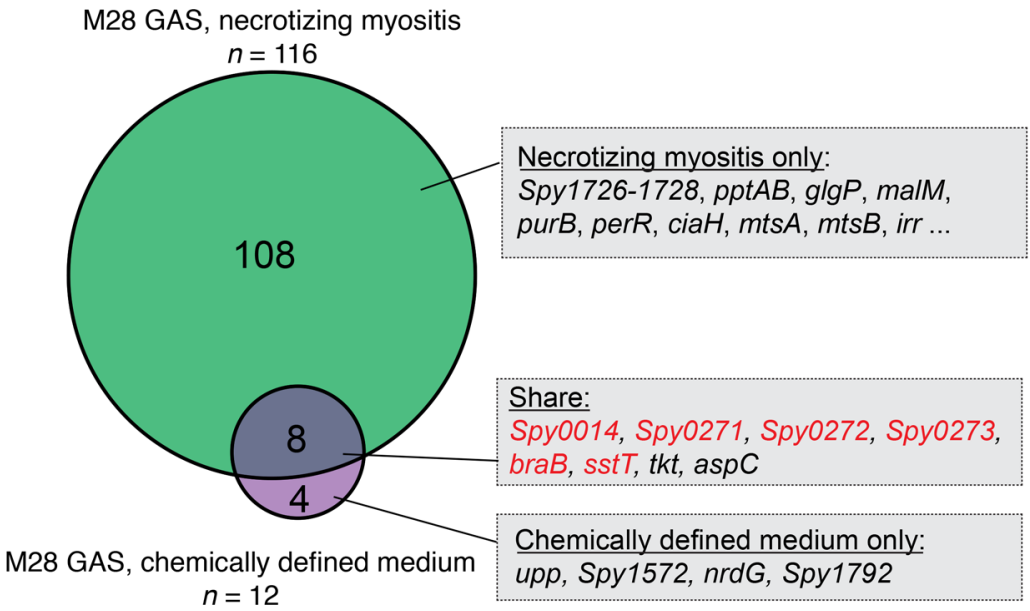

Figure 7. Overlap between GAS fitness genes required for necrotizing myositis and genes required for growth in CDM. (A) Venn diagram comparison of 126 M1 GAS genes required for NHP necrotizing myositis with 34 genes required for optimal growth in CDM ex vivo. (B) Venn diagram comparison of 116 M28 GAS genes required for necrotizing myositis with 12 genes required for growth in CDM. Genes encoding putative amino acid transporters are shown in red font for emphasis.

M1 strain MGAS2221. The $\triangle g l g P$ isogenic mutant strain is severely attenuated in its capacity to cause necrotizing myositis in NHPs, thereby confirming the TraDIS screen finding (Figure 8, A-D). We next evaluated the potential role of $g l g P$ in carbohydrate metabolism. Although the $\triangle g l g P$ mutant strain had no growth defect in medium with glucose, this mutant strain exhibited a severe growth defect when maltose or maltodextrin was provided as the sole carbohydrate in the culture medium (Figure 9, A-C). Consistent with the idea that the product of the glgP gene is involved in glycogen and starch utilization, bacteria grown in THY supplemented with starch showed evidence of starch accumulation in the isogenic mutant strain $\triangle g l g P$, but not the WT parental strain that retained the ability to metabolize starch (Figure 9D). Interestingly, in $E$. coli, glycogen accumulation was also significantly higher in $g l g P$ deletion mutants (71).

Expression of the GAS genes implicated in in vivo fitness genes during NHP necrotizing myositis. In the aggregate, data from the in vivo transposon mutant library screens and analysis of the isogenic mutant strains imply that the genes identified are expressed

in NHP necrotizing myositis. To test this finding, we generated isogenic mutant strain $\triangle p p t A B$ by deleting the pptAB genes in serotype M1 parental strain MGAS2221. Relative to the WT parental strain, the $\triangle p p t A B$ mutant strain was significantly attenuated in ability to cause necrotizing myositis in NHPs (Figure 8, A-D). PptAB has been reported as being required for exporting the SHP2 and SHP3 quorum-sensing peptides (68). However, Rgg2, the transcriptional regulator that controls expression of the $\operatorname{sh} 2$ and $\operatorname{sh} 33$ genes $(69,70)$, was not identified as important for NHP infections in our TraDIS screens. These results suggest the attenuation of the virulence phenotype in the $\triangle p p t A B$ mutant strain is likely not associated with the SHP2 and SHP3 quorum-sensing pathway in serotype M1 and M28 strains in this infection model.

Confirmation of the virulence role of glgP, a gene involved in carbohydrate utilization. Our TraDIS screens identified many GAS genes implicated in transport of nutrients, such as amino acids, vitamins, and carbohydrates. We next studied a gene likely to be involved in carbohydrate utilization. The GAS gene $g l g P$ was identified as essential for necrotizing myositis in the TraDIS screens conducted with both the serotype M1 and M28 transposon mutant libraries (Supplemental Table 4). However, glgP has not previously been implicated in GAS virulence; glgP encodes an inferred protein with homology to E. coli glycogen phosphorylase (71). We generated isogenic mutant strain $\triangle g l g P$ by deleting the $g l g P$ gene in serotype during NHP necrotizing myositis. To directly test for expression in vivo, we used TaqMan qRT-PCR to measure the transcript levels of GAS transporter genes Spy0014, Spy0271, braB, Spy1726, and pptA and metabolic gene $g l g P$ in NHP muscle tissue infected with M1 GAS MGAS2221. The transcripts of all 6 of the GAS fitness genes studied were detectable by TaqMan qRT-PCR, confirming that these genes are expressed in vivo in NHP necrotizing myositis (Figure 10A).

Expression of fitness genes in vivo in a human with necrotizing myositis. We next tested the hypothesis that the 6 targeted genes of interest are expressed in a human patient with necrotizing myositis caused by an emm 89 GAS strain. Necrotic skeletal muscle was studied by TaqMan qRT-PCR. Whole genome sequencing showed that the TaqMan primers and probes designed for M1 GAS could also be used for detecting transcripts of the 6 genes in this strain. The results confirmed the presence of transcripts from the 6 genes in the infected human patient (Figure 10B). It is important to note that the relative transcript levels for all genes tested were similar in the experimentally infected NHPs and the human with natural infection.

\section{Discussion}

GAS is an abundant human pathogen that is responsible for substantial human illness and economic loss worldwide. Necrotizing fasciitis and myositis caused by this organism are particularly devastating infections because they have high morbidity and 

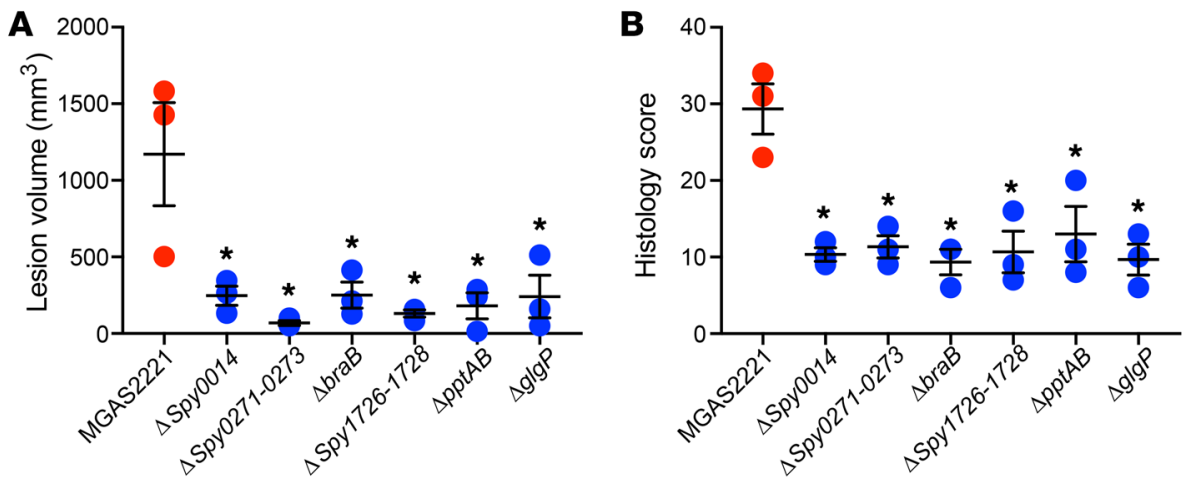

C

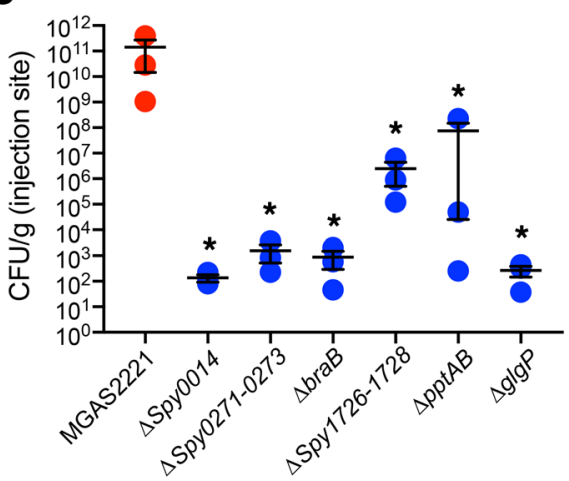

$\mathbf{E}$

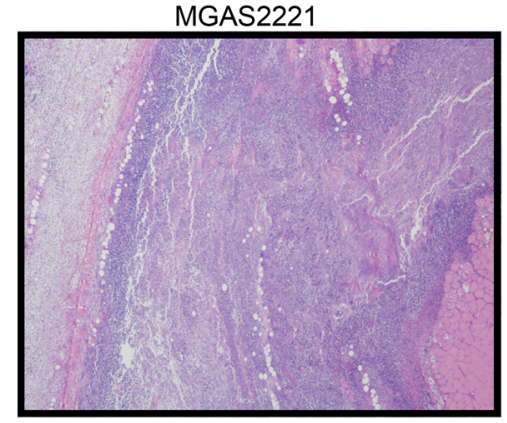

D

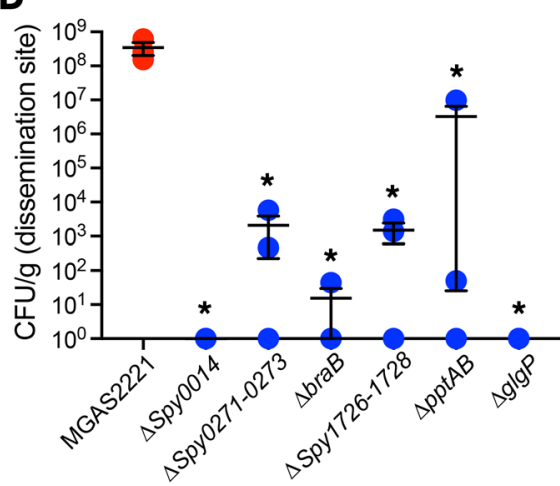

$\mathbf{F}$

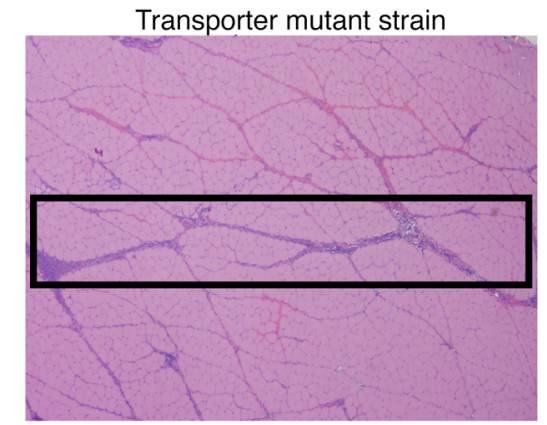

Figure 8. Virulence phenotypes of GAS isogenic transporter deletion mutant strains in NHPs. (A and B) Volume and histology score of the necrotizing myositis lesions caused by the parental WT M1 GAS strain MGAS2221 compared with each isogenic deletion mutant strain. (C and D) CFUs recovered from the inoculation site (C) and distal muscle margin (D). Replicate data $(n=3)$ are expressed as mean \pm SEM. ${ }^{*} P<0.05$, Mann-Whitney $U$ test (A, C, and D) or Wilcoxon's rank sum test (B). Micrographs of H\&E necrotizing myositis lesions caused by the parental WT stain (E) compared with a representative transporter mutant strain, $\Delta$ Spy0014 (F). The boxes enclose each necrotic lesion. Original magnification, $\times 2$. mortality. Effective treatment options for these infections remain limited, and a licensed human GAS vaccine is not available. Thus, a fuller understanding of pathogen factors that contribute to these severe diseases is warranted and may facilitate translational research activities.

Our genome-wide screens identified 126 M1 genes and 116 M28 genes contributing to fitness in NHP necrotizing myositis. Of particular importance, we discovered a substantial overlap between the genes identified in the M1 and M28 in vivo fitness screens, with 72 genes common to both serotypes, representing $57 \%$ and $64 \%$ of the M1 and M28 fitness genes, respectively. The similarity between M1 and M28 in vivo fitness gene results suggests the existence of conserved programs used by multiple diverse GAS strains to proliferate and damage tissue in necrotizing myositis. Many of the shared 72 genes encoded proven or putative metabolic enzymes implicated in complex carbohydrate metabolism (malM and $g l g P$ ), pyruvate metabolism ( $a c o A, a c o B, a c o L)$, amino acid biosynthesis (tkt, $\operatorname{aroD}, \operatorname{gln} A$, and $\operatorname{arc} B$ ), and nucleotide biosynthesis (purA and purB), suggesting these pathways are critical for GAS fitness in the environment of deep-tissue infec- tion. In addition to metabolic genes, several previously identified GAS virulence or fitness factors were also among the 72 genes. For example, ScfAB (2 putative membrane proteins) was identified as important for GAS fitness and virulence during subcutaneous infection in mice (30), and $a d c A B C$ (zinc importer) was critical for GAS virulence in mice and has vaccine interest (51).

In contrast with the similar critical gene requirements for serotype M1 and M28 GAS during experimental NHP necrotizing myositis, there was relatively little overlap between M1 GAS genes required for necrotizing myositis and growth in human saliva ex vivo (Figure 3), that is, the spectrum of genes contributing to fitness in these 2 environments was largely distinct. For example, genes encoding multiple amino acid transporters (e.g., Spy0014, braB, and sst T) required for GAS fitness during necrotizing myositis were not identified as important for growth ex vivo in human saliva (Figure $3 \mathrm{~A}$ ). In contrast, a GAS phosphate transporter encoded by the pst operon was essential for persistence in human saliva ex vivo, but was apparently dispensable for NHP muscle infection (Figure 3A). These results suggest that amino acid uptake is critical for GAS fitness during muscle 
A
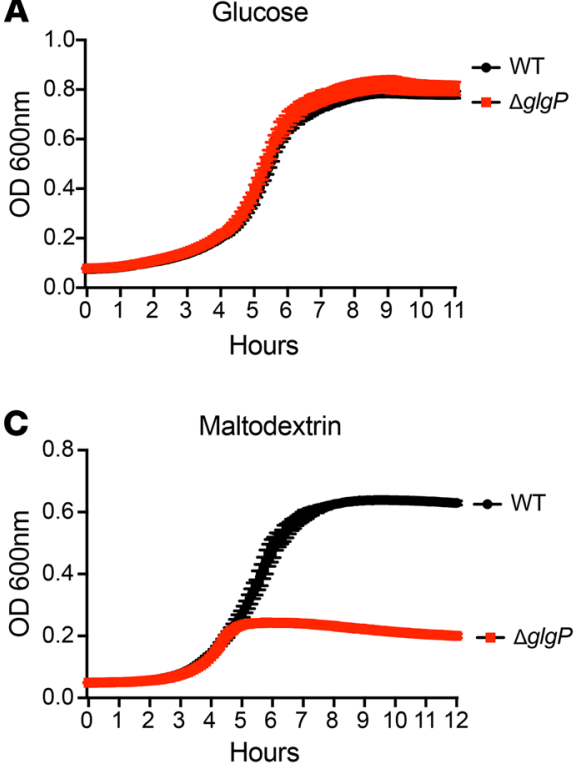

B

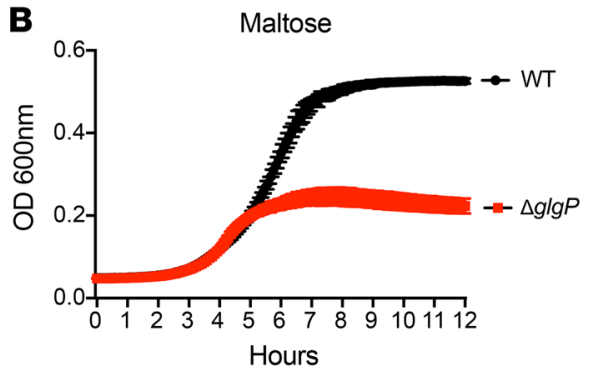

D

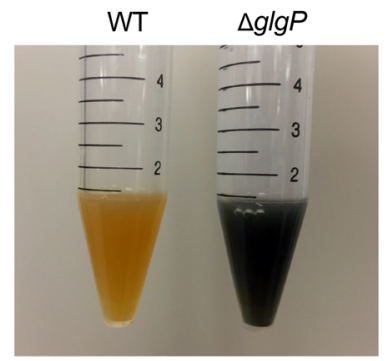

Figure 9. In vitro phenotype of isogenic mutant strain $\Delta \boldsymbol{g} \mathbf{g} \boldsymbol{P}$. Growth of WT and isogenic mutant $\triangle g l g P$ in culture medium with glucose as the sole carbohydrate source (A), medium with maltose as the sole carbohydrate source (B), and medium with maltodextrin as the sole carbohydrate source (C). (D) Accumulation of starch by the $\Delta g l g P$ mutant strain. The compositions of media with different carbohydrate sources are listed in Supplemental Table 16. infections, whereas phosphate uptake is essential for growth in human saliva ex vivo. Similarly, GAS genes contributing to NHP necrotizing myositis and mouse subcutaneous infection also had relatively little overlap (Figure 3B). Many GAS metabolic genes are specifically required for NHP necrotizing myositis. For example, genes for de novo purine nucleotide biosynthesis (purA and purB), carbohydrate utilization ( $g \lg P$ and malM), and arginine and citrulline catabolism $(\operatorname{arcABCD})$ are uniquely important for NHP necrotizing myositis. Moreover, several known streptococcal virulence-modulating factors were also identified in NHP necrotizing myositis. These included genes for GAS lipoprotein processing (lgt and $l s p)$ (72-74) and genes for a 2-component regulatory system that was essential for GAS to evade human innate immunity (ihk and irr) (75) (Figure 3B). Taken together, these results suggest the nutritional environment and the survival pressures present in the infected NHP skeletal muscle are distinct from those in human saliva ex vivo and in a mouse subcutaneous infection model. These results imply that complex gene programs used by GAS to cause other types of human infections (e.g., puerperal sepsis and pharyngitis) are also likely to be niche specific.

A key theme of our M1 and M28 NHP genome-wide screens was identification of many genes encoding transporters that are required during necrotizing myositis. Pinpointing exactly which of the transporters contribute to bacterial virulence during necrotizing myositis sheds new light on the mechanisms of GAS-host interactions in this severe infection. We identified 13 distinct transporters that were required in both M1 and M28 GAS strains. Six of these transporters were inferred to function in amino acid transport. This observation suggests the ability to efficiently acquire host amino acids is critical for the pathogenesis of GAS necrotizing myositis. In vitro growth assays showed that amino acid transporter mutant strains $\triangle S p y 0014, \triangle b r a B$, and $\triangle S p y 0271$ 0273 had a substantial growth defect in the peptide-free CDM that was ameliorated by supplementing CDM with tryptone, a source of abundant peptides. These results suggest that efficient amino acid uptake is critical for WT GAS growth when the peptide source is limited and that the nutritional environment of the infected muscle is probably a poor source of available peptides. GAS is auxotrophic for 15 amino acids considered essential for growth (65). We hypothesized that transporters Spy0014, BraB, and Spy02710273 are required for the highly efficient uptake of certain essential amino acids. As anticipated, we showed that supplementing $\mathrm{CDM}$ with methionine, histidine, and valine restored the growth of mutant strains $\triangle S p y 0271-0273, \Delta S p y 0014$, and $\Delta b r a B$, respectively, indicating these 3 transporters contribute to transport of these 3 amino acids. Interestingly, the concentrations of free methionine, histidine, and valine in the human skeletal muscle tissue are 16.4 $\mathrm{mg} / \mathrm{l}, 57.4 \mathrm{mg} / \mathrm{l}$, and $30.5 \mathrm{mg} / \mathrm{l}$, values lower than those present in CDM $(100 \mathrm{mg} / \mathrm{l})(69,76)$. In the aggregate, our results suggest efficient uptake of essential host amino acids, such as methionine, histidine, and valine, is important for GAS to cause necrotizing myositis in NHPs. The qRT-PCR data demonstrating the presence of transcripts from these transporter genes document that they are expressed in NHPs and infected humans. Together, this implies that blocking the uptake of essential amino acids by GAS might be a feasible strategy for controlling GAS infection pathology, but further studies are required to test this idea.

Since certain amino acid transporter genes crucial in necrotizing myositis are also essential for GAS growth in CDM (Figure 6), we systematically studied GAS genes required for growth in CDM. Using TraDIS, we identified 34 and 12 genes important for optimal growth in CDM in the M1 and M28 GAS strains, respectively (Figure 7). We found that a common set of 6 genes for amino acid transporters was important for both M1 and M28 GAS strains in CDM (Figure 7). Importantly, these 6 genes required for CDM growth also contributed to GAS fitness during necrotizing myositis. Collectively, our results imply that efficient acquisition of amino acids is essential for GAS to cause necrotizing myositis.

We identified several virulence-related transporters with unclear functions. One example is the putative ABC transporter comprising Spy1726 (transporter permease protein), Spy1727 (ATP-binding protein), and Spy1728 (substrate-binding lipopro- 
A

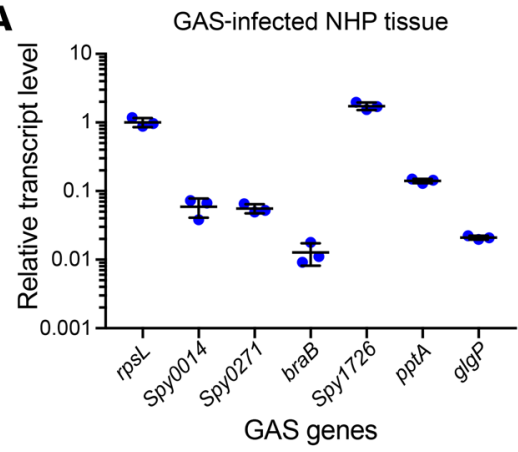

B

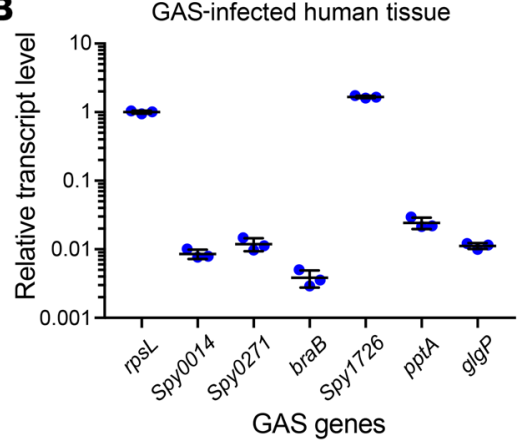

Figure 10. Relative transcript level of GAS fitness

genes. Transcript levels are shown in an infected NHP with experimental necrotizing myositis ( $(\mathbf{A})$ and in an infected human with necrotizing fasciitis (B). In vivo transcript level of GAS genes Spy0014, Spy0271, braB, Spy1726, pptA, and $g l g P$ relative to housekeeping gene $r p s L$. The experiment was performed in triplicate, and mean $\pm \mathrm{SD}$ are shown. tein). The TraDIS screen results and the virulence phenotype of the isogenic mutant strain indicated that this $A B C$ transporter of unknown function is critical for the ability of GAS to cause NHP skeletal muscle pathology. Although its function is not known, multiple additional leads indicated ABC transporter Spy17261728 plays a role in host-pathogen interactions. For example, the Spy1726-1728 operon is upregulated when GAS cells are in human blood and macrophages $(77,78)$. In addition, Spy1726-1728 is regulated by the CovR/S 2-component system, a global virulence gene regulator in GAS (79). Proteomic studies show that the substrate-binding lipoprotein Spy1726 is located on the bacterial cell surface and thus might be a candidate for vaccine or other translational research (67). Future structural and functional studies on this transporter appear to be warranted.

Genes encoding certain secreted or surface-located GAS virulence factors were not identified as essential for necrotizing myositis in our genome-wide screens. Notable examples include the speB gene encoding the secreted protease virulence factor SpeB and the emm gene encoding M protein. Many previous investigations have demonstrated $\mathrm{SpeB}$ as contributing significantly to GAS virulence in mouse and NHP models of necrotizing myositis $(8,80)$. Our screen results suggest that the loss of SpeB production resulted in a neutral (M28 GAS) or increased fitness (M1 GAS) phenotype during NHP necrotizing myositis. We speculate that SpeB-negative GAS cells can be transcomplemented in situ by SpeB-producing GAS cells concurrently present at the infection site. Since SpeB-producing cells at the infection site could potentially protect SpeB-negative GAS and production of SpeB is energy consuming, loss of SpeB may not be deleterious to GAS fitness under the conditions tested. M protein is a major GAS virulence factor with antiphagocytic activities $(81,82)$. M has been shown to contribute to GAS virulence in a murine model of soft tissue infection (7). However, our screens did not identify M protein as essential for GAS fitness during necrotizing myositis. It may be that the selective pressures at work in the NHP skeletal muscle in vivo are different from those present in mouse soft tissue. The role of $\mathrm{M}$ protein in GAS virulence during necrotizing myositis remains to be determined.

To summarize, in this work, we used 2 distinct transposon mutant libraries made in serotypes of GAS that cause abundant human cases of severe invasive infections to identify genes contributing to GAS fitness in an NHP model of necrotizing myositis. NHP infection using 6 isogenic mutant strains confirmed the crucial requirement for the genes identified by our TraDIS screens.
Our findings complement work conducted with other transposon mutant screens that identified GAS genes contributing to fitness during growth in vitro, collected human saliva and human blood ex vivo, and mouse subcutaneous infection. The findings presented herein may ultimately lead to better ways to diagnose, treat, and prevent necrotizing myositis and fasciitis caused by GAS, infections with devastating consequences to the human host.

\section{Methods}

Bacterial strains. Strain MGAS2221 is genetically representative of the pandemic clone of serotype M1 GAS that arose in the 1980s and has spread worldwide (45). Strain MGAS27961 is genetically representative of a virulent serotype M28 clone that is prevalent in the United States and elsewhere (46). These 2 strains have WT alleles of all major transcriptional regulators known to affect virulence, such as $\operatorname{cov} R$ and covs, $\operatorname{rop} B, m g a$, and $\operatorname{roc} A$.

GAS strain growth conditions. The GAS strains were cultured in Todd-Hewitt broth supplemented with $0.5 \%$ yeast extract (THY). When required, GAS strains were grown in a chemically defined medium (CDM). The CDM was made according to previous descriptions with modifications $(69,83,84)$. The composition of the CDM is presented in Supplemental Table 11. Amino acids were added to the designated concentration. For growth in medium with a carbohydrate source other than glucose, GAS strains were cultured in maltose medium and maltodextrin medium. The composition of these media is presented in Supplemental Table 16.

Transposon mutant libraries and culture conditions. The mutant library stock generated in serotype M1 strain MGAS2221 using transposon plasmid pGh9:ISS1 was recently described $(29,47)$. The serotype M28 strain MGAS27961 transposon mutant library stock was made by essentially identical methods. The strains were grown in Todd Hewitt broth supplemented with $0.2 \%$ yeast extract (THY) at $37^{\circ} \mathrm{C}$ with $5 \% \mathrm{CO}_{2}$.

Preparation of transposon mutant library frozen stock for NHP infection. Transposon mutant library stock ( $100 \mu \mathrm{l}$; M1 or M28 GAS library) was inoculated in $500 \mathrm{ml}$ THY supplemented with $0.5 \mu \mathrm{g} / \mathrm{ml}$ erythromycin and cultured at $37^{\circ} \mathrm{C}$ for 8 hours. The proliferated transposon library stock was pelleted by centrifugation, washed 3 times with saline, and then suspended in $10 \mathrm{ml}$ saline supplemented with $20 \%$ glycerol. The suspended mutant library stock was aliquoted into cryogenic tubes and stored at $-80^{\circ} \mathrm{C}$ until subsequent use in NHP infections.

NHP necrotizing myositis infection model used for TraDIS analysis. A well-described NHP model of necrotizing myositis was used (43). For the transposon mutant library screens, 6 cynomolgus macaques (1-3 
years, $2-4 \mathrm{~kg}$, males and females) each were used for the serotype M1 and M28 screens. Briefly, NHPs were sedated and bacteria were inoculated in the right quadriceps. Animals were observed and necropsied 24 hours after infection. To analyze the output transposon insertion library stock, an approximately $0.5 \mathrm{~g}(\sim 0.5-1.0 \mathrm{~cm}$ diameter) biopsy of necrotic muscle was obtained from the inoculation site, homogenized in $1 \mathrm{ml}$ sterile PBS, transferred to $40 \mathrm{ml}$ Todd Hewitt broth, and incubated for 6 hours. Before incubation, $100 \mu \mathrm{l}$ was removed from the 40 $\mathrm{ml}$ culture, serially diluted in sterile PBS, and plated to determine the number of CFUs in the output library stock. Infected tissue was also collected for histologic examination. The inoculum used for the input M1 transposon mutant library was approximately $5 \times 10^{8} \mathrm{CFU} / \mathrm{kg}$. The inoculum used for the input M28 transposon mutant library was $1 \times 10^{10} \mathrm{CFU} / \mathrm{kg}$. A higher dose of the M28 input transposon mutant library was used because, in previous NHP studies, an approximately 10- to 100-fold higher inoculum of M28 strains compared with M1 strains was needed to generate similar disease character.

Screen for GAS genes required for growth in CDM, a peptide-free chemically defined medium. $50 \mu \mathrm{l}$ of the M1 or M28 GAS transposon mutant library stock suspended in PBS was inoculated into 4 Falcon tubes, each containing $40 \mathrm{ml}$ CDM. The amount of inoculum used for the input mutant library stock was approximately $10^{7} \mathrm{CFU} /$ tube. The 4 inoculated tubes constituted 4 biological replicates for the CDM screen assay. Immediately after inoculation, the tubes were vortexed thoroughly, and $200 \mu \mathrm{l}$ of the inoculated CDM from each of the 4 replicate cultures was plated onto 4 THY agar plates and incubated at $37^{\circ} \mathrm{C}$ for 12 hours. GAS cells growing on the plates were harvested and represent the composition of the input mutant pools ( 0 hours mutant pools, $n=4)$. The remaining inoculated $\mathrm{CDM}$ was incubated at $37^{\circ} \mathrm{C}$ for 12 hours. After 12 hours incubation, $200 \mu \mathrm{l}$ of the CDM culture from each of the 4 replicate cultures was plated onto THY agar plates to recover output mutant pools ( 12 hours mutant pools, $n=4)$. The collected mutant pools were stored at $-80^{\circ} \mathrm{C}$ for TraDIS analysis.

DNA preparation and massively parallel sequencing. The mutant library genomic DNA preparation and DNA sequencing were performed according to procedures described previously for TraDIS analysis (29, 47). The PCR-amplified libraries were sequenced with a NextSeq550 instrument (Illumina) using a single-end 75-cycle protocol.

Processing of TraDIS sequencing reads and data analysis. The processing of TraDIS reads and data analysis were performed according to previously described procedures (29). Briefly, the multiplexed raw Illumina reads obtained from the input and output mutant pools were parsed with FASTX Barcode Splitter (http://hannonlab.cshl.edu/fastx_toolkit/ commandline.html). The resulting sequencing reads were analyzed with the TraDIS toolkit (50), and tradis_comparison. R was used to compare the reads mapped per gene between the input pools and the output pools. The GAS genes with significantly changed mutant frequency ( $\log _{2}$ fold change greater than $+/-1 ; q<0.01$ ) in the output mutant pools were interpreted as contributing to GAS fitness. For both necrotizing myositis screen and CDM screen, Illumina sequencing reads of the M1 and M28 GAS input and output libraries were deposited in the NCBI's Sequence Read Archive (SRA BioProject PRJNA494385).

Construction and characterization of isogenic mutant strains. Isogenic mutant strains were derived from WT parental strain MGAS2221, the organism used for construction of the serotype M1 transposon mutant library. Primers used for generating the mutant strains are listed in Supplemental Table 10. Markerless isogenic mutant strains were constructed by nonpolar deletion of the target gene(s) using allelic exchange (43). For example, to delete Spy0014, primer sets 0014-1/2 and 0014-3/4 were used to amplify 2 approximately $1.5 \mathrm{~kb}$ fragments flanking Spy0014 with genomic DNA purified from serotype M1 strain MGAS2221. The 2 flanking fragments were combined by overlapextension PCR with primers 0014-1 and 0014-4. The combined fragment was cloned into suicide vector pBBL740 and transformed into parental strain MGAS2221. The plasmid integrant was used for allelic exchange, as described previously (43). PCR was used to identify potential mutant candidates containing the desired deletion. All other isogenic mutant strains were generated using analogous methods. Whole genome sequencing of all isogenic mutant strains was done to confirm the absence of spurious mutations.

Infection of NHPs with isogenic mutant strains. To confirm the role of candidate genes in necrotizing myositis molecular pathogenesis and thereby validate the TraDIS data, the virulence of the parental WT strain MGAS2221 and the 6 isogenic deletion-mutant strains was assessed in the NHP necrotizing myositis infection model. Animals randomly assigned to different strain treatment groups received $10^{8} \mathrm{CFU} / \mathrm{kg}$ of one strain (WT or isogenic mutant) in the right limb and a different strain in the left limb. Each strain was tested in triplicate. The animals were observed continuously and necropsied at 24 hours after inoculation.

Histopathology analysis. For histology evaluation, lesions were excised and visually inspected. Lesions (necrotic muscle) were measured in 3 dimensions, and volume was calculated using the formula for an ellipsoid. Tissue taken from the inoculation site was trisected, fixed in $10 \%$ phosphate-buffered formalin, and embedded in paraffin using standard automated instruments. Histology of the 3 sections taken from each limb was scored by a pathologist blinded to the strain treatment groups, as described previously (43). To obtain the quantitative CFU data, diseased muscle obtained from the inoculation site or distal hip margin was weighed and homogenized (Omni International) in $1 \mathrm{ml} \mathrm{PBS}$; CFUs were determined by plating serial dilutions of the homogenate. Statistical differences between strain groups were determined using the Mann-Whitney $U$ test.

Iodine staining of WT and $\triangle g l g P$ mutant strain. The WT strain and isogenic $\triangle g l g P$ mutant strain were cultured for 8 hours in $10 \mathrm{ml}$ of THY supplemented with $2 \mathrm{~g} / \mathrm{l}$ of soluble starch (Sigma-Aldrich). GAS cells were pelleted and washed 5 times with saline to remove the culture medium. After the final saline wash, GAS cells were suspended in 1 $\mathrm{ml}$ of saline. Gram's iodine solution $(10 \mu \mathrm{l})$ was added to the cell suspensions to visualize glycogen accumulation in the GAS cell. Only the $\triangle g l g P$ mutant strain displayed a dark blue iodine stain phenotype.

Isolation of total RNA from GAS-infected NHP quadriceps muscle sections and skeletal muscle from a human with GAS necrotizing fasciitis. Infected tissue from NHPs or human patients was stored at $-80^{\circ} \mathrm{C}$ in DNA/RNA Shield (Zymo Research) or RNAlater (Invitrogen), respectively, thawed on ice, transferred to a tube containing $2 \mathrm{ml}$ of cold TE, and diluted with either $2 \mathrm{ml}$ or $1.3 \mathrm{ml} 2 \times$ DNA/RNA Shield. Tissue samples were homogenized with an Omni TH homogenizer (Omni International). Prior to lysis, supernatants were divided into either 4 aliquots, each containing $900 \mu$ l, or 3 aliquots, each containing $950 \mu \mathrm{l}$, for NHP or human samples, respectively. The tissues were lysed by ballistic disintegration using a FastPrep-96 instrument (MP Biomedicals) and Zymo tubes containing 0.1 and $0.5 \mathrm{~mm}$ ZR BashingBeads (Zymo Research). Lysis was repeated 3 times at 1,600 rpm for 1 minute, and tubes were placed on ice for 1 minute after each lysis step. Particulate 
matter present in the supernatants was eliminated with QIAshredder homogenizers (QIAGEN). RNA was isolated using the Zymobiomics RNA Kit (Zymo Research) following the manufacturer's instructions, with the following exceptions: all aliquots from a particular sample were pooled together before passing them through the first column, and the recommended DNase treatment was performed twice for the human samples. Total RNA quality was assessed with an RNA Nanochip and an Agilent 2100 Bioanalyzer (Agilent Technologies).

qRT-PCR analysis of infected NHP and human muscle. Total RNA extracted from infected NHP or human skeletal muscle was converted into cDNA using SuperScript III Reverse Transcriptase, random primers, RNaseOUT, and dNTPs (all from Invitrogen). qRT-PCR was performed using TaqMan Fast Universal PCR Master Mix (Applied Biosystems) with an ABI 7500 Fast System instrument (Life Technologies). The genes tested were Spy0014, Spy0271, braB, Spy1726, pptA, and $\lg g$. The sequences of primers and probes used in the qRT-PCR experiments are listed in Supplemental Table 17. Each experiment was performed with 3 technical replicates at 3 different dilutions. Transcript levels were normalized relative to the $r p s L$ gene (encoding $30 \mathrm{~S}$ ribosomal protein S12).

Statistics. Results of lesion volume and CFU recovery from NHPs are expressed as mean \pm SEM, with statistically significant differences determined using the Mann-Whitney $U$ test (Prism 6, Graphpad Software). Results of histology scoring of infected NHP muscle are expressed as mean \pm SEM, with statistically significant differences determined using Wilcoxon's rank sum test (Prism 6). Nonparametric tests were used because the data were shown to not follow a normal distribution using the Shapiro-Wilk test (Prism 6). A $P$ value of less than 0.05 was considered statistically significant.

Study approval. All animal experiments were approved by the Institutional Animal Care and Use Committee of Houston Methodist Research Institute (protocol AUP-1217-0058). The human tissue was collected as part of a study approved by the Institutional Review Board at Houston Methodist Research Institute (protocol 0907-0151). The subject gave informed consent.

\section{Author contributions}

LZ performed and analyzed TraDIS experiments, constructed and characterized isogenic mutant strains, and wrote the manuscript. RJO planned and conducted experiments involving the NHPs, analyzed the resulting data, and wrote the manuscript. SBB analyzed the genomic data and wrote the manuscript. JME contributed critical discussions about transporter physiology and performed the TaqMan qRT-PCR to measure the transcript levels of GAS transporter genes in infected NHP and human muscle. MOS performed the genome sequencing of the isogenic mutant strains and provided technical support for the NHP studies. SLK constructed and characterized isogenic mutant strains. CCC provided extensive technical support for all phases of the study. ARLC and ASW provided intellectual guidance for TraDIS data analysis. LJ oversaw and performed the NHP experiments. JMM designed the studies, analyzed experiments, wrote the manuscript, and oversaw the project.

\section{Acknowledgments}

This work was supported by funds from the Fondren Foundation (to JMM). ARLC is supported by the University of Cambridge Doctoral Training Partnership scheme, which is funded by the Biotechnology and Biological Sciences Research Council, United Kingdom (reference 1503883). We thank Frank DeLeo, Magnus Gottsfredsson, Karl G. Kristinsson, David M. Morens, and Kathryn E. Stockbauer for critical reading of the manuscript and suggesting improvements. We are indebted to Lillian S. Kao for providing tissue specimens from a patient with necrotizing myositis. We thank Annessa Smith and Caroline White for superb veterinary technical assistance.

Address correspondence to: James M. Musser, Department of Pathology and Genomic Medicine, Houston Methodist Research Institute, Houston, Texas 77030, USA. Phone: 713.441.5890; Email: jmmusser@houstonmethodist.org.
1. Stevens DL, Bryant AE. Necrotizing soft-tissue infections. N Engl JMed. 2017;377(23):2253-2265.

2. Misiakos EP, Bagias G, Patapis P, Sotiropoulos D, Kanavidis P, Machairas A. Current concepts in the management of necrotizing fasciitis. Front Surg. 2014;1:36.

3. Nelson GE, et al. Epidemiology of invasive group A streptococcal infections in the United States, 2005-2012. Clin Infect Dis. 2016;63(4):478-486.

4. Lepoutre A, et al. Epidemiology of invasive Streptococcus pyogenes infections in France in 2007. J Clin Microbiol. 2011;49(12):4094-4100.

5. Carapetis JR, Steer AC, Mulholland EK, Weber M. The global burden of group A streptococcal diseases. Lancet Infect Dis. 2005;5(11):685-694.

6. Cunningham MW. Pathogenesis of group A streptococcal infections. Clin Microbiol Rev. 2000;13(3):470-511.

7. Ashbaugh CD, Warren HB, Carey VJ, Wessels MR. Molecular analysis of the role of the group A streptococcal cysteine protease, hyaluronic acid capsule, and M protein in a murine model of human invasive soft-tissue infection. J Clin Invest. 1998;102(3):550-560.
8. Olsen RJ, et al. Decreased necrotizing fasciitis capacity caused by a single nucleotide mutation that alters a multiple gene virulence axis. Proc Natl Acad Sci U S A . 2010;107(2):888-893.

9. Wessels MR, Moses AE, Goldberg JB, DiCesare TJ. Hyaluronic acid capsule is a virulence factor for mucoid group A streptococci. Proc Natl Acad Sci U S A. 1991;88(19):8317-8321.

10. Zhu H, Liu M, Sumby P, Lei B. The secreted esterase of group a streptococcus is important for invasive skin infection and dissemination in mice. Infect Immun. 2009;77(12):5225-5232.

11. Bricker AL, Carey VJ, Wessels MR. Role of NADase in virulence in experimental invasive group A streptococcal infection. Infect Immun. 2005;73(10):6562-6566.

12. Limbago B, Penumalli V, Weinrick B, Scott JR. Role of streptolysin $\mathrm{O}$ in a mouse model of invasive group A streptococcal disease. Infect Immun. 2000;68(11):6384-6390.

13. Zhu L, Olsen RJ, Lee JD, Porter AR, DeLeo FR, Musser JM. Contribution of secreted NADase and streptolysin $\mathrm{O}$ to the pathogenesis of epidemic serotype M1 Streptococcus pyogenes infections. Am J Pathol. 2017;187(3):605-613.

14. Ferretti JJ, et al. Complete genome sequence of an M1 strain of Streptococcus pyogenes. Proc Natl Acad Sci U S A. 2001;98(8):4658-4663.

15. Kizy AE, Neely MN. First Streptococcus pyogenes signature-tagged mutagenesis screen identifies novel virulence determinants. Infect Immun. 2009;77(5):1854-1865.

16. Armbruster CE, et al. Genome-wide transposon mutagenesis of Proteus mirabilis: Essential genes, fitness factors for catheter-associated urinary tract infection, and the impact of polymicrobial infection on fitness requirements. PLoS Pathog. 2017;13(6):e1006434.

17. Gawronski JD, Wong SM, Giannoukos G, Ward DV, Akerley BJ. Tracking insertion mutants within libraries by deep sequencing and a genome-wide screen for Haemophilus genes required in the lung. Proc Natl Acad Sci US A. 2009;106(38):16422-16427.

18. Subashchandrabose S, Smith SN, Spurbeck RR, Kole MM, Mobley HL. Genome-wide detection of fitness genes in uropathogenic Escherichia coli during systemic infection. PLoS Pathog. 
2013;9(12):e1003788.

19. Wang N, Ozer EA, Mandel MJ, Hauser AR. Genome-wide identification of Acinetobacter baumannii genes necessary for persistence in the lung. MBio. 2014;5(3):e01163-e01114.

20. Weerdenburg EM, et al. Genome-wide transposon mutagenesis indicates that Mycobacterium marinum customizes its virulence mechanisms for survival and replication in different hosts. Infect Immun. 2015;83(5):1778-1788.

21. Le Breton Y, et al. Genome-wide identification of genes required for fitness of group $\mathrm{A}$ streptococcus in human blood. Infect Immun . 2013;81(3):862-875.

22. Lau GW, et al. A functional genomic analysis of type 3 Streptococcus pneumoniae virulence. Mol Microbiol. 2001;40(3):555-571.

23. Zhang $X$, et al. RNA-seq and Tn-seq reveal fitness determinants of vancomycin-resistant Enterococcus faecium during growth in human serum. BMC Genomics. 2017;18(1):893.

24. Shames SR, Liu L, Havey JC, Schofield WB, Goodman AL, Roy CR. Multiple Legionella pneumophila effector virulence phenotypes revealed through high-throughput analysis of targeted mutant libraries. Proc Natl Acad Sci U S A. 2017;114(48):E10446-E10454.

25. Gutierrez MG, Yoder-Himes DR, Warawa JM. Comprehensive identification of virulence factors required for respiratory melioidosis using Tn-seq mutagenesis. Front Cell Infect Microbiol. 2015;5:78.

26. Palace SG, Proulx MK, Lu S, Baker RE, Goguen JD. Genome-wide mutant fitness profiling identifies nutritional requirements for optimal growth of Yersinia pestis in deep tissue. MBio. 2014;5(4):e01385-14.

27. McCarthy AJ, Stabler RA, Taylor PW. Genomewide identification by transposon insertion sequencing of Escherichia coli K1 genes essential for in vitro growth, gastrointestinal colonizing capacity, and survival in serum. J Bacteriol. 2018;200(7):e00698-17.

28 . Wilde $\mathrm{AD}$, et al. Bacterial hypoxic responses revealed as critical determinants of the host-pathogen outcome by TnSeq analysis of Staphylococcus aureus invasive infection. PLoS Pathog. 2015;11(12):e1005341.

29. Zhu L, Charbonneau ARL, Waller AS, Olsen RJ, Beres SB, Musser JM. Novel genes required for the fitness of Streptococcus pyogenes in human saliva. mSphere. 2017;2(6):e00460-17.

30. Le Breton Y, et al. Genome-wide discovery of novel M1T1 group A streptococcal determinants important for fitness and virulence during soft-tissue infection. PLoS Pathog. 2017;13(8):e1006584.

31. Sun $\mathrm{H}$, et al. Plasminogen is a critical host pathogenicity factor for group A streptococcal infection. Science. 2004;305(5688):1283-1286.

32. Sun H, Wang X, Degen JL, Ginsburg D. Reduced thrombin generation increases host susceptibility to group A streptococcal infection. Blood. 2009;113(6):1358-1364.

33. Kasper KJ, et al. Bacterial superantigens promote acute nasopharyngeal infection by Streptococcus pyogenes in a human MHC Class II-dependent manner. PLoS Pathog. 2014;10(5):e1004155.
34. Sriskandan S, et al. Enhanced susceptibility to superantigen-associated streptococcal sepsis in human leukocyte antigen-DQ transgenic mice. J Infect Dis. 2001;184(2):166-173.

35. Marcum JA, Kline DL. Species specificity of streptokinase. Comp Biochem Physiol, B. 1983;75(3):389-394.

36. Wulf RJ, Mertz ET. Studies on plasminogen. 8. Species specificity of streptokinase. Can J Biochem. 1969;47(10):927-931.

37. Langridge GC, et al. Simultaneous assay of every Salmonella Typhi gene using one million transposon mutants. Genome Res. 2009;19(12):2308-2316.

38. van Opijnen T, Camilli A. Transposon insertion sequencing: a new tool for systems-level analysis of microorganisms. Nat Rev Microbiol. 2013;11(7):435-442.

39. Naseer U, Steinbakk M, Blystad H, Caugant DA. Epidemiology of invasive group A streptococcal infections in Norway 2010-2014: A retrospective cohort study. Eur JClin Microbiol Infect Dis. 2016;35(10):1639-1648.

40. Smit PW, Lindholm L, Lyytikäinen O, Jalava J, Pätäri-Sampo A, Vuopio J. Epidemiology and emm types of invasive group A streptococcal infections in Finland, 2008-2013. Eur J Clin Microbiol Infect Dis. 2015;34(10):2131-2136.

41. Meehan M, Murchan S, Gavin PJ, Drew RJ, Cunney R. Epidemiology of an upsurge of invasive group A streptococcal infections in Ireland, 2012-2015. J Infect. 2018;77(3):183-190.

42. Smeesters PR, Laho D, Beall B, Steer AC, Van Beneden CA. Seasonal, geographic, and temporal trends of emm clusters associated with invasive group A Streptococcal infections in US multistate surveillance. Clin Infect Dis. 2017;64(5):694-695.

43. Zhu L, et al. A molecular trigger for intercontinental epidemics of group A streptococcus. J Clin Invest. 2015;125(9):3545-3559.

44. Sumby P, et al. Evolutionary origin and emergence of a highly successful clone of serotype M1 group a streptococcus involved multiple horizontal gene transfer events. J Infect Dis. 2005;192(5): 771-782.

45. Nasser W, et al. Evolutionary pathway to increased virulence and epidemic group $\mathrm{A}$ streptococcus disease derived from 3,615 genome sequences. Proc Natl Acad Sci U S A. 2014;111(17):E1768-E1776.

46. Kachroo P, et al. Integrated analysis of population genomics, transcriptomics and virulence provides novel insights into Streptococcus pyogenes virulence. Nat Genet. In press. https://doi. org/10.1038/s41588-018-0343-1.

47. Charbonneau ARL, et al. Defining the ABC of gene essentiality in streptococci. BMC Genomics. 2017;18(1):426.

48. Abel S, Abel zur Wiesch P, Davis BM, Waldor MK. Analysis of bottlenecks in experimental models of infection. PLoS Pathog. 2015;11(6):e1004823.

49. Le Breton $Y$, et al. Essential genes in the core genome of the human pathogen Streptococcus pyogenes. Sci Rep. 2015;5:9838.

50. Barquist L, et al. The TraDIS toolkit: sequencing and analysis for dense transposon mutant libraries. Bioinformatics. 2016;32(7):1109-1111.
51. Makthal N, et al. A critical role of zinc importer AdcABC in group A streptococcus-host interactions during infection and its implications for vaccine development. EBioMedicine. 2017;21:131-141.

52. van Sorge NM, et al. The classical lancefield antigen of group a streptococcus is a virulence determinant with implications for vaccine design. Cell Host Microbe. 2014;15(6):729-740.

53. Henningham A, et al. Virulence role of the GlcNAc side chain of the Lancefield cell wall carbohydrate antigen in non-M1-serotype group A streptococcus. MBio. 2018;9(1):e02294-17.

54. Honda-Ogawa M, et al. Streptococcus pyogenes endopeptidase $\mathrm{O}$ contributes to evasion from complement-mediated bacteriolysis via binding to human complement factor C1q.J Biol Chem. 2017;292(10):4244-4254.

55. Brouwer S, et al. Endopeptidase PepO regulates the SpeB cysteine protease and is essential for the virulence of invasive M1T1 Streptococcus pyogenes. J Bacteriol. 2018;200(8):e00654-17.

56. Reid SD, et al. Characterization of an extracellular virulence factor made by group A streptococcus with homology to the Listeria monocytogenes internalin family of proteins. Infect Immun. 2003;71(12):7043-7052.

57. Brenot A, King KY, Caparon MG. The PerR regulon in peroxide resistance and virulence of Streptococcus pyogenes. Mol Microbiol. 2005;55(1):221-234.

58. Treviño J, Liu Z, Cao TN, Ramirez-Peña E, Sumby P. RivR is a negative regulator of virulence factor expression in group A streptococcus. Infect Immun. 2013;81(1):364-372.

59. Biswas I, Scott JR. Identification of rocA, a positive regulator of covR expression in the group A streptococcus. J Bacteriol. 2003;185(10):3081-3090.

60. Lynskey NN, et al. RocA truncation underpins hyper-encapsulation, carriage longevity and transmissibility of serotype M18 group A streptococci. PLoS Pathog. 2013;9(12):e1003842.

61. Jain I, Miller EW, Danger JL, Pflughoeft KJ, Sumby P. RocA is an accessory protein to the virulence-regulating CovRS two-component system in group A streptococcus. Infect Immun. 2017;85(11):e00274-17.

62. Miller EW, Danger JL, Ramalinga AB, Horstmann N, Shelburne SA, Sumby P. Regulatory rewiring confers serotype-specific hyper-virulence in the human pathogen group A streptococcus. Mol Microbiol. 2015;98(3):473-489.

63. Bernard PE, et al. RocA has serotype-specific gene regulatory and pathogenesis activities in serotype M28 group A streptococcus. Infect Immun. 2018;86(11):e00467-18

64. Basavanna S, et al. The effects of methionine acquisition and synthesis on Streptococcus pneumoniae growth and virulence. PLOS ONE. 2013;8(1):e49638.

65. Davies HC, Karush F, Rudd JH. Effect of amino acids on steady-state growth of a group a hemolytic Streptococcus. J Bacteriol. 1965;89:421-427.

66. Pancholi V, Caparon M. Streptococcuspyogenes metabolism. In: Ferretti JJ, Stevens DL, Fischetti VA, eds. Streptococcus pyogenes: Basic Biology to Clinical Manifestations. Oklahoma City, OK; University of Oklahoma Health Sciences Center: 2016.

67. Rodríguez-Ortega MJ, et al. Characterization 
and identification of vaccine candidate proteins through analysis of the group A streptococcus surface proteome. Nat Biotechnol. 2006;24(2):191-197.

68. Chang JC, Federle MJ. PptAB exports Rgg quorum-sensing peptides in Streptococcus. PLoS One. 2016;11(12):e0168461.

69. Chang JC, LaSarre B, Jimenez JC, Aggarwal C, Federle MJ. Two group A streptococcal peptide pheromones act through opposing Rgg regulators to control biofilm development. PLoS Pathog. 2011;7(8):e1002190.

70. LaSarre B, Chang JC, Federle MJ. Redundant group a streptococcus signaling peptides exhibit unique activation potentials. J Bacteriol. 2013;195(18):4310-4318.

71. Alonso-Casajús N, et al. Glycogen phosphorylase, the product of the glgP Gene, catalyzes glycogen breakdown by removing glucose units from the nonreducing ends in Escherichia coli. J Bacteriol. 2006;188(14):5266-5272.

72. Chimalapati S, et al. Effects of deletion of the Streptococcus pneumoniae lipoprotein diacylglyceryl transferase gene lgt on $\mathrm{ABC}$ transporter function and on growth in vivo. PLOS ONE. 2012;7(7):e41393.
73. Das S, et al. Contribution of lipoproteins and lipoprotein processing to endocarditis virulence in Streptococcus sanguinis. J Bacteriol. 2009;191(13):4166-4179.

74. Weston BF, Brenot A, Caparon MG. The metal homeostasis protein, Lsp, of Streptococcus pyogenes is necessary for acquisition of zinc and virulence. Infect Immun. 2009;77(7):2840-2848.

75. Voyich JM, et al. Engagement of the pathogen survival response used by group A streptococcus to avert destruction by innate host defense. Jimmunol. 2004;173(2):1194-1201.

76. Bergström J, Fürst P, Norée LO, Vinnars E. Intracellular free amino acid concentration in human muscle tissue. J Appl Physiol. 1974;36(6):693-697.

77. Graham MR, et al. Group A streptococcus transcriptome dynamics during growth in human blood reveals bacterial adaptive and survival strategies. Am J Pathol. 2005;166(2):455-465.

78. Hertzén E, et al. Intracellular Streptococcus pyogenes in human macrophages display an altered gene expression profile. PLOS ONE. 2012;7(4):e35218.

79. Graham MR, et al. Virulence control in group A streptococcus by a two-component gene regu- latory system: global expression profiling and in vivo infection modeling. Proc Natl Acad Sci U S A. 2002;99(21):13855-13860.

80. Do H, et al. Leaderless secreted peptide signaling molecule alters global gene expression and increases virulence of a human bacterial pathogen. Proc Natl Acad Sci U S A. 2017;114(40):E8498-E8507.

81. Horstmann RD, Sievertsen HJ, Knobloch J, Fischetti VA. Antiphagocytic activity of streptococcal $\mathrm{M}$ protein: selective binding of complement control protein factor H. Proc Natl Acad Sci U S A. 1988;85(5):1657-1661.

82. Perez-Casal J, Caparon MG, Scott JR. Introduction of the emm6 gene into an emm-deleted strain of Streptococcus pyogenes restores its ability to resist phagocytosis. Res Microbiol. 1992;143(6):549-558.

83. Pérez-Pascual D, et al. RovS and its associated signaling peptide form a cell-to-cell communication system required for Streptococcus agalactiae pathogenesis. MBio. 2015;6(1):e02306-14.

84. van de Rijn I, Kessler RE. Growth characteristics of group A streptococci in a new chemically defined medium. Infect Immun. 1980;27(2):444-448. 